\title{
Maximum Circuit Activity Estimation Using Pseudo-Boolean Satisfiability
}

\author{
Hratch Mangassarian, Student Member, IEEE, Andreas Veneris, Senior Member, IEEE, \\ and Farid N. Najm, Fellow, IEEE
}

\begin{abstract}
With lower supply voltages, increased integration densities and higher operating frequencies, power grid verification has become a crucial step in the VLSI design cycle. The accurate estimation of maximum instantaneous power dissipation aims at finding the worst-case scenario where excessive simultaneous switching could impose extreme current demands on the power grid. This problem is highly input-pattern dependent and is proven to be NP-hard. In this work, we capitalize on the compelling advancements in satisfiability solvers to propose a pseudo-Boolean satisfiability-based framework that reports the input patterns maximizing circuit activity, and consequently peak dynamic power, in combinational and sequential circuits. The proposed framework is enhanced to handle unit gate delays and output glitches. In order to disallow unrealistic input transitions, we show how to integrate input constraints in the formulation. Finally, a number of optimization techniques, such as the use of gate switching equivalence classes, are described to improve the scalability of the proposed method. An extensive suite of experiments on ISCAS85 and ISCAS89 circuits confirms the robustness of the approach compared to simulation-based techniques and encourages further research for low-power solutions using Boolean satisfiability.
\end{abstract}

Index Terms-Maximum Circuit Activity, Peak Dynamic Power, SAT, Pseudo-Boolean Satisfiability.

\section{INTRODUCTION}

Lower supply voltages, increased integration densities, and higher operating frequencies, among other factors, are producing devices that are more sensitive to power dissipation and reliability problems $[1,2]$. Excessive power dissipation can lead to overheating, electromigration and a reduced chip lifetime [3]. Also, large instantaneous power consumption causes voltage drop and ground bounce, resulting in circuit delays and soft errors [4]. Therefore, accurate power estimation during the design phase is crucial to avoid a time-consuming redesign process and in the worst-case an extremely costly tape-out failure [3]. As a result, reliability analysis has steadily become a critical part of the design process of digital circuits.

In CMOS circuits, power dissipation depends on the extent of circuit switching activity, which is input pattern dependent.

Copyright (c) 2011 IEEE. Personal use of this material is permitted. However, permission to use this material for any other purposes must be obtained from the IEEE by sending an email to pubs-permissions@ieee.org.

H. Mangassarian is with the Department of Electrical and Computer Engineering, University of Toronto, Toronto, ON, Canada, M5S 3G4 (hratch@eecg.toronto.edu).

A. Veneris is with the Department of Electrical and Computer Engineering and with the Department of Computer Science, University of Toronto, Toronto, ON, Canada, M5S 3G4 (veneris@eecg.toronto.edu).

F. N. Najm is with the Department of Electrical and Computer Engineering, University of Toronto, Toronto, ON, Canada, M5S 3G4 (f.najm@utoronto.ca).
The maximum circuit activity estimation problem aims at finding the input patterns which cause peak instantaneous dynamic power: A worst-case scenario where excessive simultaneous gate switching imposes extreme current demands on the power grid [1], leading to unwanted voltage drops. This problem is NP-complete for combinational circuits, and PSPACE-complete for sequential circuits [5].

Existing methods for maximum activity estimation can be classified into the two general categories of simulationbased and non-simulative approaches [6]. The former rely on extensive circuit simulations under representative input vectors. On the other hand, non-simulative approaches use characteristics of the circuit and stochastic properties of input vectors to perform power estimation without explicit circuit simulation [6].

In this work, we leverage the advancements and ongoing research in satisfiability-based solvers $[19,20,22]$ to tackle this problem using a symbolic approach. Boolean satisfiability (SAT) solvers and their extensions, such as quantified Boolean formula satisfiability (QBF) solvers and pseudo-Boolean satisfiability (PBS) solvers, have become attractive tools for solving theoretically intractable problems in VLSI CAD, in areas such as testing [21], verification [25] and physical design [27]. Furthermore, any improvement to the state-of-theart in satisfiability solving translates into an immediate benefit to all satisfiability-based solutions.

A pseudo-Boolean satisfiability-based framework is presented for generating tight lower bounds on maximum weighted circuit activity within a clock-cycle [17]. The described framework is applicable to both combinational and sequential circuits, and to both zero and unit gate delay models. Glitches are accounted for in the unit gate delay formulation. As a formal method, our technique may be less scalable than simulation-based frameworks. For this reason, it is intended as a complementary, rather than an alternative, approach to simulations, that can discover "hidden" activity corner-cases. Additionally, the proposed method is not applicable in the presence of variability or uncertainty in gate delays.

In order to disallow unrealistic input transitions and invalid initial states, we show how to integrate input constraints in the problem formulation. Several optimization techniques are presented to improve the scalability of the proposed method. In particular, switching equivalence classes are used to group gates that are most likely to switch in tandem, thus reducing the symbolic problem size.

An extensive suite of experiments on ISCAS85 and ISCAS89 circuits confirms the effectiveness of SAT in a low- 
power analysis application. Coupled with the modeling flexibility offered by SAT and its extensions, this encourages further research in the use of satisfiability-based tools as platforms to solve other low-power problems.

The paper is organized as follows. Section II summarizes previous work. Section III presents background on SAT and PBS. Section IV briefly discusses assumptions and preliminaries. Section V gives the pseudo-Boolean satisfiability formulations for the maximum activity problem in combinational and sequential circuits. Section VI extends the framework to handle glitches due to gate delays. Section VII describes how to apply input constraints to disallow unrealistic input transitions. Section VIII discusses optimizations and heuristics. Section IX shows experimental results and Section $\mathrm{X}$ concludes the paper.

\section{PREVIOUS WORK}

A number of techniques have been proposed in the literature to estimate the maximum peak power dissipation [9-15] or the maximum instantaneous current $[4,7,8]$ of a CMOS circuit. In [4, 7], a loose upper bound on the maximum instantaneous current is generated in linear time by propagating signal uncertainties. This bound is subsequently tightened using a branch-and-bound algorithm that considers spatial signal correlations. Extending the characterization of signal correlations from $[4,7]$ and the authors in [8] exploit mutually exclusive gate switching to generate tighter upper bounds. However, for larger circuits, the gap between the generated upper bounds and lower bounds obtained using simulations can remain considerable.

In [9], the authors present an Automatic Test Pattern Generation (ATPG) based greedy algorithm that attempts to maximize fanout-weighted gate flips. They also provide a statistical quality measure for the generated lower bounds on maximum circuit activity. In [10], the method is extended to cover sequential circuits as well as glitches. A continuous optimization method is set forward in [11], which treats the Boolean input space as a real-valued vector space and makes use of a gradient based heuristic to estimate the maximum power.

The authors in [12] use genetic algorithms to compare the effect of different delay models on peak power. They conclude that peak power estimated using a zero-delay model is inaccurate, whereas peak power estimated using a unit-delay model is reasonably accurate. In [13], various genetic spotoptimization heuristics are employed to avoid local maxima during the search for maximum single-cycle activities using a variable delay model. [13] reports significant improvements over simulations. Both [12] and [13] are able to handle larger circuits and more general delay models than symbolic approaches such as ours. However, unlike symbolic techniques, they are unable to prove the optimality of their results.

The work presented in [14] considers $n$ independent and identically distributed samples of power-per-cycle. Drawing on the theory of asymptotic extreme order statistics, they model the largest of the $n$ sample values using a Weibull distribution. They then perform a maximum likelihood estimation of the largest power value. The approach of [6] is an extension of [14], which handles more corner cases and uses different statistical distributions. For example, instead of a Weibullmaxima model, [6] uses the so-called Beta-exceedances model. Both of these methods are considered simulative approaches, and are therefore highly input-pattern dependent and lack the exhaustive property of symbolic techniques. However, they can handle any delay model and they scale better than symbolic techniques.

The approach that is closest to this work is given in [15], where the power dissipation of a circuit is modeled as a multi-output Boolean function in terms of the primary inputs. A disjoint cover enumeration as well as a branchand-bound algorithm are used to maximize the number of weighted gate transitions. An approximation strategy for upper bounding maximum power is also proposed. However, the described techniques can become computationally expensive. Furthermore, sequential circuits are not covered.

The work in [16], published after our original paper [17], proposes the use of temporal and spatial windows in order to split the original maximum activity estimation problem into smaller, more manageable subproblems. They present a high-level algorithm for using symbolic simulation to create a symbolic network, which is then translated to a pseudoBoolean optimization problem. They do not describe the construction of this symbolic network in detail. The addition of spatial and temporal restrictions on the optimization problem in [16] is orthogonal to our work, and provides a viable method to scale activity estimation techniques, including the approach described in this paper. It should be noted that our work [17] was the first to propose such a PBO-based approach to activity estimation.

\section{BACKGROUND}

\section{A. Boolean Satisfiability}

A propositional logic formula $\Phi$ can be constructed over a set of Boolean variables using Boolean connectives such as - (negation), $\wedge$ (conjunction) and $\vee$ (disjunction). $\Phi$ is said to be satisfiable or SAT if it has a satisfying assignment: a truth assignment to each of its variables that causes it to evaluate to 1 . Otherwise, $\Phi$ is said to be unsatisfiable or UNSAT. The problem of Boolean satisfiability consists of determining whether $\Phi$ is SAT. In modern SAT solvers, the logic formula $\Phi$ is given in Conjunctive Normal Form (CNF) as a conjunction of clauses where each clause is a disjunction of literals. A literal is an instance of a variable or its negation. In order for a formula to be SAT, at least one literal in each clause must evaluate to 1 . For example, the CNF formula given in (1) is $\operatorname{SAT}$ since $\left\{x_{1}=1, x_{2}=0, x_{3}=1\right\}$ is a satisfying assignment.

$$
\Phi=\left(x_{1} \vee x_{2}\right) \wedge\left(x_{1} \vee \bar{x}_{2} \vee \bar{x}_{3}\right) \wedge\left(x_{3}\right)
$$

A logic circuit can be converted to a CNF formula in linear time under reasonable assumptions [21], such that there is a one-to-one correspondence between the variables of the generated CNF formula and the gates of the corresponding circuit, and such that satisfying variable assignments in the CNF formula correspond to valid gate output values in the circuit. 
As such, a circuit and its corresponding SAT formulation are often referred to interchangeably in this paper.

Modern SAT solvers implement Conflict-Driven Clause Learning (CDCL) [30]. They are able to solve large industrial SAT problems with millions of variables and clauses. During the search, SAT solvers prune parts of the search-space that do not contain satisfying assignments by analyzing their decisions and learning conflict clauses. For example, consider the CNF formula in (1) and suppose that the solver has made the unsatisfiable variable assignments $\left\{x_{1}=0, x_{2}=1, x_{3}=1\right\}$. A conflict is generated and analyzed by the solver, which realizes that $\left\{x_{1}=0\right\}$ cannot be extended to a satisfying assignment, and therefore adds the conflict clause $\left(x_{1}\right)$ to $\Phi$ in order to force $\left\{x_{1}=1\right\}$.

\section{B. Pseudo-Boolean Satisfiability}

A pseudo-Boolean constraint over Boolean variables $x_{0}, x_{1}, \ldots, x_{n-1}$ is an inequality of the form:

$$
\sum_{i=0}^{n-1} c_{i} \cdot l_{i} \geq c_{n}
$$

where $c_{i} \in \mathbb{Z}$ and $l_{i}$ is a literal corresponding to $x_{i}$, i.e. $l_{i}=x_{i}$ or $l_{i}=\bar{x}_{i}$. Note that a CNF clause is a special case of a pseudo-Boolean constraint with $c_{i}=0$ or 1 , and $c_{n}=1$. A pseudo-Boolean constraint becomes satisfied if (2) holds.

A pseudo-Boolean formula $\Psi$ is a conjunction of pseudoBoolean constraints. The problem of pseudo-Boolean satisfiability (PBS) questions the existence of a truth assignment to $x_{0}, x_{1}, \ldots, x_{n-1}$ satisfying all the pseudo-Boolean constraints in $\Psi$. A pseudo-Boolean optimization (PBO) problem tries to find a satisfiable assignment to a PBS problem $\Psi$ that also minimizes a given objective function:

$$
\mathcal{F}(\mathbf{x})=\sum_{i=0}^{n-1} d_{i} \cdot l_{i}
$$

where $\mathbf{x}=\left\langle x_{0}, \ldots, x_{n-1}\right\rangle$ and $d_{i} \in \mathbb{Z}$.

For example, given $\Psi$ and $\mathcal{F}$ as shown in (4) below, both $\left\{x_{1}=1, x_{2}=0, x_{3}=1\right\}$ and $\left\{x_{1}=1, x_{2}=0, x_{3}=0\right\}$ are satisfying assignments. However, the former minimizes $\mathcal{F}$.

$$
\begin{aligned}
\Psi & =\left(2 x_{1}-3 x_{2} \geq 1\right) \wedge\left(x_{1}+x_{2}+\bar{x}_{3} \geq 1\right) \\
\mathcal{F} & =\bar{x}_{3}-x_{1}+2 \bar{x}_{2}
\end{aligned}
$$

The classical approach for solving combinatorial optimization problems, including PBO, has historically been branchand-bound [31]. In general, these algorithms are able to prune the search tree by using estimates on the value of the optimization function. [31] gives an overview of branch-and-bound techniques for PBO. Motivated by recent advances in SAT solvers, the most effective SAT techniques, including clause learning, lazy data structures and conflict-driven branching heuristics, have been extended to PBO [32]. In this work, we use the PBO solver MINISAT+ [22] which translates pseudoBoolean constraints to SAT and runs a state-of-the-art SAT solver [20] on the produced SAT instance. The latter approach is particularly suited to problems consisting of mostly SAT clauses and relatively few pseudo-Boolean constraints [22], which is the case in this work. Furthermore, any advancements in SAT solving directly enhances such a strategy.

In MINISAT+, the objective function is minimized using a linear search. MINISAT+ first runs the SAT solver without considering $\mathcal{F}(\mathbf{x})$ in order to get an initial SAT solution $\mathbf{x}_{\mathbf{0}}$, with $\mathcal{F}\left(\mathbf{x}_{\mathbf{0}}\right)=k$, where $k$ is the corresponding initial value of the objective function. The new pseudo-Boolean constraint $\mathcal{F}(\mathbf{x}) \leq k-1$ is subsequently added to the original problem. The SAT solver is then run on the updated CNF formula and this process is repeated until the problem becomes UNSAT. The solution corresponding to the last $k$ before the problem becomes UNSAT is the optimal solution minimizing the objective function.

\section{ASSUMPTIONS AND PRELIMINARIES}

Flip-flop-controlled synchronous digital circuits are considered. Primary inputs and flip-flop (DFF) outputs can only switch at the beginning of the clock-cycle. This assumption is considered valid in related previous work as well.

The dynamic power dissipation of a CMOS circuit during a clock-cycle can be approximated as follows:

$$
P=\frac{1}{2} V_{d d}^{2} \sum_{i=1}^{m} C_{i} \cdot f_{i}
$$

where $m$ is the number of circuit gates, $C_{i}$ is the capacitive load on gate $g_{i}$ and $f_{i}$ is the output transition count of $g_{i}$ during a clock-cycle. Under the assumption that the clock period is sufficiently small, it is sound to interpret (5) as the instantaneous dynamic power during that clock-cycle [9-15]. In the remainder of this work, the terms circuit activity and switched capacitance refer to the summation in (5) and are used interchangeably.

The following notation is used throughout this paper. $T$ represents a combinational or sequential circuit and $\mathcal{G}(T)$ denotes the set of gates in $T$ excluding primary inputs and states. Symbol $m$ denotes the number of gates in $\mathcal{G}(T)$. Symbols $x$ and $s$ are the Boolean vectors respectively denoting the primary inputs and state elements (DFFs) of a sequential circuit $T$. Variables $x_{i}$ and $s_{i}$ denote the $i$ th primary input and state element of $T$. Variable $g_{i}$ refers to $i$ th gate in $T$ and can assume all basic gate types, such as AND, OR, XOR, NOT and BUFFER.

Circuit unrolling consists of replicating the combinational component of a sequential design and connecting the nextstate of each time-frame to the current-state of the following time-frame. As will be described later, this process allows the PBO solver to reason on the operation of a sequential circuit. A superscripted variable (e.g. $g^{j}$ ) denotes the copy of that variable in the $j$ th copy of the unrolled circuit $T . s^{0}$ denotes the initial-state of $T$. FANOUTS $\left(g_{i}\right)$ (FANINS $\left(g_{i}\right)$ ) denotes the set of fanouts (fanins) of $g_{i}$. Finally, in all the examples, it is assumed that $C_{i}=\mid$ FANOUTS $\left(g_{i}\right) \mid$ for internal gates and $C_{i}=1$ for primary output gates. 


\section{Zero-Delay Maximum Activity Computation USING PBO}

\section{A. Maximum Activity for Combinational Circuits}

Under a zero-delay model, each gate transition count $f_{i}$ is a Boolean variable because $g_{i}$ can flip at most once per clockcycle. Accordingly, the summation in (5) can be rewritten as:

$$
\sum_{i=1}^{m} C_{i} \cdot\left(g_{i}\left(x^{0}\right) \oplus g_{i}\left(x^{1}\right)\right)
$$

where $x^{0}$ and $x^{1}$ are consecutively applied primary input vectors and $g_{i}(x)$ denotes the steady-state value of gate $g_{i}$ given primary input vector $x$.

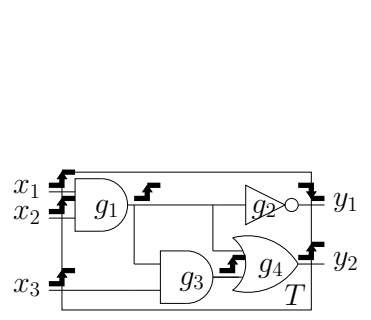

(a) Zero-delay gate switching

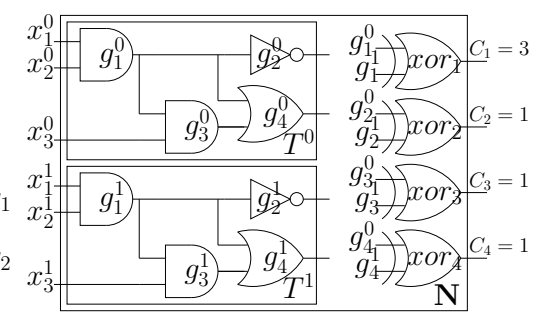

(b) Zero-delay PBO formulation
Fig. 1. Zero-delay PBO formulation for combinational circuits

One needs to find the pair of consecutive primary input vectors $\left\langle x^{0^{*}}, x^{1^{*}}\right\rangle$ maximizing (6). This problem is formulated as a PBO problem as follows. A new circuit $\mathbf{N}$ is constructed which contains two replicas of the original circuit $T$, named $T^{0}$ and $T^{1}$. The primary input vector $x^{0}\left(x^{1}\right)$ is applied to $T^{0}$ $\left(T^{1}\right)$. Next, every pair of corresponding gates, $g_{i}^{0}$ in $T^{0}$ and $g_{i}^{1}$ in $T^{1}$, is fed to a new XOR gate, called $x_{0} r_{i}$, in N. Clearly, the output of each xor $_{i}$ yields $g_{i}\left(x^{0}\right) \oplus g_{i}\left(x^{1}\right)$. Fig. 1(b) shows the construction of $\mathbf{N}$ for the circuit given in Fig. 1(a).

Let $\mathrm{CNF}(\mathbf{N})$ denote the translation of $\mathbf{N}$ into $\mathrm{CNF}$ clauses (which are also pseudo-Boolean constraints). Clearly, the solution of the following PBO problem maximizes the value of (6):

$$
\begin{aligned}
\Psi & =\operatorname{CNF}(\mathbf{N}) \\
\mathcal{F} & =-\sum_{i=1}^{m} C_{i} \cdot \text { xor }_{i}
\end{aligned}
$$

Note that only the target function $\mathcal{F}$ is not already given as a set of clauses. The pseudo-Boolean formula $\Psi$, which is simply the CNF of $\mathbf{N}$, markedly suits the choice of the non-native (SAT-based) PBO solver MINISAT+ [22], since the latter is only left to translate $\mathcal{F}$ into $\mathrm{CNF}$.

Example 1 Consider the original circuit $T$ and the corresponding construction $\mathbf{N}$ shown in Fig. 1. Disregarding primary input flips, an optimal solution to the associated $\mathrm{PBO}$ problem is $\left\langle x^{0^{*}}, x^{1^{*}}\right\rangle=\langle\langle 0,0,0\rangle,\langle 1,1,1\rangle\rangle$, which amounts to a total switched capacitance of 6 units by flipping all four gate outputs as shown in Fig. 1(a).

\section{B. Maximum Activity for Sequential Circuits}

Let $g_{i}\left(s^{0}, x\right)$ denote the steady-state value of gate $g_{i}$ given initial-state $s^{0}$ and primary input vector $x$. For a sequential circuit, the transition count $f_{i}$ depends on both primary input transitions and the initial state. Therefore, estimating the peak power per cycle for sequential circuits is equivalent to finding a triplet $\left\langle s^{0^{*}}, x^{0^{*}}, x^{1^{*}}\right\rangle$ consisting of an initial state $s^{0}$ and consecutive primary input vectors, $x^{0}$ and $x^{1}$, that maximizes the following summation:

$$
\sum_{i=1}^{m} C_{i} \cdot\left(g_{i}\left(s^{0}, x^{0}\right) \oplus g_{i}\left(s^{1}, x^{1}\right)\right)
$$

where $s^{1}$ denotes the next-state of the circuit.

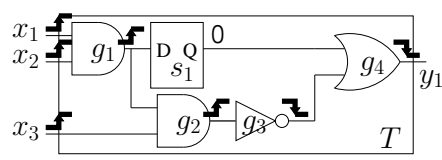

(a) Zero-delay switching

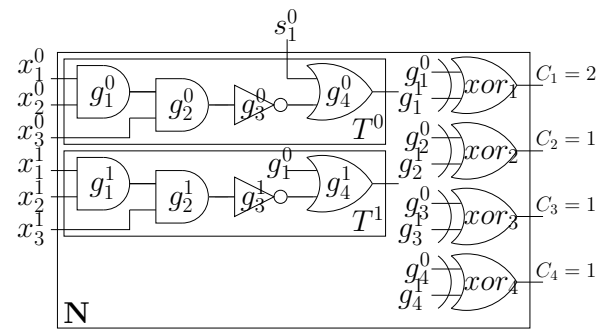

(b) Zero-delay PBO formulation

Fig. 2. Zero-delay PBO formulation for sequential circuits

Finding this triplet is formulated as a PBO problem as follows. First, DFF inputs (outputs) are transformed into circuit pseudo-outputs (pseudo-inputs). A new circuit $\mathbf{N}$ is constructed which contains two replicas of this full-scanned circuit. Moreover, the pseudo-outputs of the first time-frame $T^{0}$ are connected to the corresponding pseudo-inputs of the second time-frame $T^{1}$. This two time-frame iterative logic array expansion of the original sequential circuit is referred to as circuit unrolling. Next, similarly to the combinational case, every pair of corresponding gates, $g_{i}^{0}$ in $T^{0}$ and $g_{i}^{1}$ in $T^{1}$, is fed to a new XOR gate. Clearly, the output of each $x_{i}$ yields $g_{i}\left(s^{0}, x^{0}\right) \oplus g_{i}\left(s^{1}, x^{1}\right)$. Fig. 2(b) shows the construction of $\mathbf{N}$ for the sequential circuit given in Fig. 2(a). Note that in this example, $s_{1}^{1}=g_{1}^{0}$.

The resulting PBO problem can be expressed by the set of equations in (7), using the above description of the circuit $\mathbf{N}$.

Example 2 Consider the circuit $T$ and the corresponding $\mathbf{N}$ shown in Fig. 2. Not counting flips at DFF outputs $\left(s_{1}\right)$ or primary inputs, an optimal solution to the $\mathrm{PBO}$ problem for sequential circuits given in this section is $\left\langle s^{0^{*}}, x^{0^{*}}, x^{1^{*}}\right\rangle=$ $\langle\langle 0\rangle,\langle 0,0,0\rangle,\langle 1,1,1\rangle\rangle$, which amounts to a total switched capacitance of 5 units as shown in Fig. 2(a). However, this solution might be suboptimal if gate delays are considered. Section VI describes how delay is integrated into the PBO problem. 
The given problem formulation allows for any initial state and primary input transitions to be returned in the optimal solution. In Section VII, we describe how to add constraints to the PBS problem to disallow certain initial states, as well as illegal or unlikely combinations of primary inputs.

\section{Modeling Delay}

Different input signal arrival times might cause a gate to flip several times during one clock-cycle. In fact, glitches due to gate propagation delays can dominate the maximum instantaneous power in some cases $[10,12]$. On the other hand, empirical results in [12] show that a unit gate delay model yields reasonably accurate power estimates. This section discusses the integration of unit gate delay into the problem formulation. It is also explained how this can be extended to arbitrary (but fixed) delay using a preprocessing step.

What follows is applicable to sequential circuits with no combinational loops (sequential loops are obviously allowed), in order to avoid unstable and metastable signals. In other terms, the full-scanned version of the sequential circuit is a Directed Acyclic Graph (DAG). For each DAG node $n_{i} \in$ $\{x, s, \mathcal{G}(T)\}$, we define its max-level $L\left(n_{i}\right)$ and min-level $l\left(n_{i}\right)$ as follows:

\section{Definition 1}

$$
L\left(n_{i}\right)= \begin{cases}\max _{\left\{n_{j} \in \operatorname{FANINS}\left(n_{i}\right)\right\}} L\left(n_{j}\right)+1 & \text { if } n_{i} \in \mathcal{G}(T) \\ 0 & \text { if } n_{i} \in\{x, s\}\end{cases}
$$

\section{Definition 2}

$$
l\left(n_{i}\right)= \begin{cases}\min _{\left\{n_{j} \in \text { FANINS }\left(n_{i}\right)\right\}} l\left(n_{j}\right)+1 & \text { if } n_{i} \in \mathcal{G}(T) \\ 0 & \text { if } n_{i} \in\{x, s\}\end{cases}
$$

$L\left(g_{i}\right)$ and $l\left(g_{i}\right)$ essentially denote the lengths of, respectively, the longest and shortest simple paths to gate $g_{i}$, in terms of number of gates, starting from a primary input in $x$ or a pseudo-input in $s$. Let $\mathcal{L}=\max _{g_{i} \in \mathcal{G}} L\left(g_{i}\right)$ designate the largest max-level in the circuit. Under a unit-delay model, time $t$ is a discrete variable, meaningful in $\{0, \ldots, \mathcal{L}\}$. Moreover, the signal arrival time at the output of gate $g_{i}$ following a certain path from a primary input or a $D F F$ is equal to the length of the traveled path to gate $g_{i}$.

Let $\mathcal{G}_{t}$ describe the set of all gates whose max-levels and min-levels bound $t$ inclusively.

\section{Definition $3 \mathcal{G}_{t}=\left\{g_{i} \in \mathcal{G} \mid l\left(g_{i}\right) \leq t \leq L\left(g_{i}\right)\right\}$}

If some gate $g_{i}$ does not belong to $\mathcal{G}_{t}$, then either $l\left(g_{i}\right)>t$ or $L\left(g_{i}\right)<t$. The former implies that the shortest signal arrival time from an input or a pseudo-input to a fanin of $g_{i}$ takes at least $t$ time-steps. So $g_{i}$ can only flip strictly after time-step $t$. Similarly, the latter implies that $g_{i}$ can only flip strictly before time-step $t$. Therefore, any gate that could potentially flip at time-step $t$ belongs to $\mathcal{G}_{t}$.

Consider a circuit whose gate logic values have stabilized given initial state $s^{0}$ and primary input vector $x^{0}$. In a unitdelay framework where each gate requires one time-step to switch, this is equivalent to applying $s^{0}$ and $x^{0}$ at $t=-1$. The primary input vector $x^{1}$ is applied at the start of a new clockcycle at $t=0$, and we let $g_{i}^{t}\left(s^{0}, x^{0}, x^{1}\right)$ denote the value of gate $g_{i}$ at time-step $t$. Note that the output value of $g_{i}$ depends on both $x^{0}$ and $x^{1}$ because if $t<l\left(g_{i}\right), g_{i}^{t}\left(s^{0}, x^{0}, x^{1}\right)=$ $g_{i}\left(s^{0}, x^{0}\right)$, which is defined in Section V-B. Accordingly, the total switched capacitance can be given by:

$$
\sum_{t=1}^{\mathcal{L}} \sum_{g_{i} \in \mathcal{G}_{t}} C_{i} \cdot\left(g_{i}^{t-1}\left(s^{0}, x^{0}, x^{1}\right) \oplus g_{i}^{t}\left(s^{0}, x^{0}, x^{1}\right)\right)
$$

The inner summation in (9) adds the capacitances of the gates whose outputs flip at time $t$. This summation only checks gates in $\mathcal{G}_{t}$ and disregards all other gates, because only the gates in $\mathcal{G}_{t}$ can potentially flip at time-step $t$. The outer summation adds the total switched capacitances across timesteps $t=1$ to $\mathcal{L}$.

To maximize (9), we will again construct a new circuit $\mathbf{N}$ that will be used by the PBO solver. In order to do so, we need to create an XOR gate for each term in the summation of (9), representing each potential glitch. As such, we need to store the value of each gate at only time-steps when its output value may potentially flip. The remainder of this section describes and proves the correctness of a circuit construction $\mathbf{N}$ that "remembers" all gate flips in $T$.

This construction is illustrated with the use of an example. Consider the sequential circuit $T$ shown in Fig. 2(a). First, DFF inputs (outputs) are transformed into circuit pseudo-outputs (pseudo-inputs). The min-level and max-level of each node can be calculated in linear time by visiting nodes in topological order starting from primary inputs and pseudo-inputs. As a result, the sets $\mathcal{G}_{1}, \mathcal{G}_{2}, \ldots, \mathcal{G}_{\mathcal{L}}$ can be generated. For the circuit in Fig. 2(a), these sets are as follows:

$$
\begin{aligned}
& \mathcal{G}_{1}=\left\{g_{1}, g_{2}, g_{4}\right\}, \mathcal{G}_{2}=\left\{g_{2}, g_{3}, g_{4}\right\}, \\
& \mathcal{G}_{3}=\left\{g_{3}, g_{4}\right\}, \mathcal{G}_{4}=\left\{g_{4}\right\} .
\end{aligned}
$$

For each time-step $t$, for $0 \leq t \leq \mathcal{L}$, we associate a timecircuit $T^{t}$ containing the following time-gates:

$$
\mathcal{G}\left(T^{t}\right)= \begin{cases}\left\{g_{i}^{t} \mid g_{i} \in \mathcal{G}_{t}\right\} & \text { if } t \geq 1 \\ \left\{g_{i}^{0} \mid g_{i} \in \mathcal{G}(T)\right\} & \text { if } t=0,\end{cases}
$$

as shown in Fig. 3. At the base case, $T^{0}$ contains all the gates in $T$, whereas $T^{t}$, for $t \geq 1$, contains every gate that can potentially switch at time $t$. The new circuit $\mathbf{N}$ in Fig. 3 accommodates all these time-circuits $T^{0}, T^{1}, \ldots, T^{\mathcal{L}}$.

Now we describe the gate interconnections in $\mathbf{N}$. The gates of $T^{0}$ are interconnected identically to the original fullscanned circuit, given pseudo-input vector $s^{0}$ and primary input vector $x^{0}$, as shown in Fig. 3. For a time-gate $g_{i}^{t}$ in timecircuit $T^{t}$, with $t \geq 1$, there are three cases for connecting it to its new inputs, depending on each fanin of the corresponding gate $g_{i}$ in the original circuit $T$ :

1) If the fanin gate was originally another internal gate, the given time-gate must be connected to the most recent corresponding time-gate strictly before the current timestep: No two time-gates in the same time-circuit can be connected because they can only change simultaneously. 


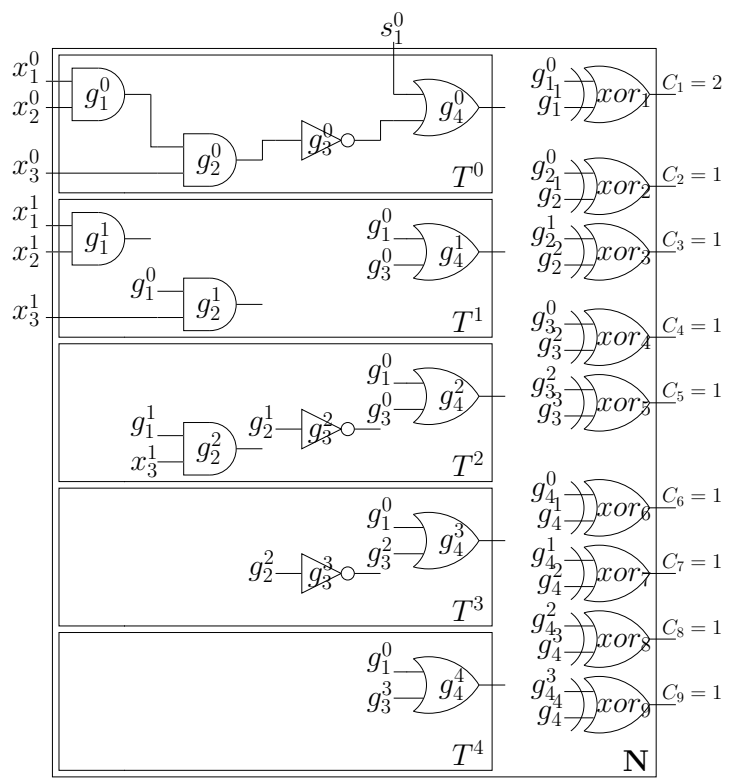

Fig. 3. Unit-delay sequential PBO formulation

2) If the fanin gate was originally a primary input, the given time-gate must be connected to the corresponding new primary input in $x^{1}$.

3) If the fanin gate was originally a $\mathrm{DFF}$ output, the given time-gate must be connected to the corresponding pseudo-output in $T^{0}$.

Formally, consider a gate $g_{i} \in \mathcal{G}(T)$, such that $\operatorname{FANIN}\left(g_{i}\right)=$ $\left\{g_{\alpha}, x_{\beta}, s_{\gamma}\right\}$, where $g_{\alpha} \in \mathcal{G}(T), x_{\beta}$ is a primary input and $s_{\gamma}$ is a DFF output. Each of these fanins corresponds to one of the three different cases above. In the new circuit $\mathbf{N}$, for each time-step $t \geq 1$ where $g_{i}^{t}$ exists, it will be connected to the following fanins:

$$
\operatorname{FANIN}\left(g_{i}^{t}\right)=\left\{g_{\alpha}^{\max \left\{j \mid g_{\alpha}^{j} \in \mathcal{G}\left(T^{j}\right), j<t\right\}}, x_{\beta}^{1}, \operatorname{FANIN}\left(s_{\gamma}\right)^{0}\right\}
$$

Here, FANIN $\left(s_{\gamma}\right)$ denotes the only fanin of $s_{\gamma}$, which is its corresponding pseudo-output, or next-state. FANIN $\left(s_{\gamma}\right)^{0}$ denotes the time-gate corresponding to this pseudo-output in $T^{0}$ of $\mathbf{N}$.

In what follows, we use the notation $g_{i} @ t$ to denote the value of gate $g_{i}$ in the original circuit $T$ at time-step $t$.

Lemma 1 Given any $s^{0}, x^{0}, x^{1}$, the time-gate $g_{i}^{t}$ in timecircuit $T^{t}$ of $\mathbf{N}$ holds the value of $g_{i} @ t$ in $T, \forall i$ and $\forall t \geq 0$.

Proof: The proof uses strong induction on the time-step variable $t$.

- Base case: At time-step $t=0$, every gate $g_{i}$ in $T$ assumes its steady-state value given initial state $s^{0}$ and primary input vector $x^{0}$. Furthermore, time-circuit $T^{0}$ in $\mathbf{N}$ is a replica of the original full-scanned circuit of $T$, with primary inputs set to $x^{0}$ and initial state $s^{0}$. As such, given any $s^{0}, x^{0}, x^{1}$, the time-gate $g_{i}^{0}$ in time-circuit $T^{0}$ of $\mathbf{N}$ holds the value of $g_{i} @ 0$ in $T, \forall i$.

- Inductive hypothesis: Given any $s^{0}, x^{0}, x^{1}$, the time-gate $g_{i}^{j}$ in time-circuit $T^{j}$ of $\mathbf{N}$ holds the value of $g_{i} @ j$ in $T$, $\forall i$ and $\forall j[0 \leq j<t]$.
- Inductive step: Consider a hypothetical gate $g_{i}$ in $T$ whose fanins cover all three cases for interconnecting time-gates in $\mathbf{N}$. In other terms, let $\operatorname{FANIN}\left(g_{i}\right)=$ $\left\{g_{\alpha}, x_{\beta}, s_{\gamma}\right\}$, where $g_{\alpha} \in \mathcal{G}(T), x_{\beta}$ is a primary input and $s_{\gamma}$ is a DFF output. As discussed, the corresponding time-gate $g_{i}^{t}$ in $T^{t}$ of $\mathbf{N}$ will have $\operatorname{FanIN}\left(g_{i}^{t}\right)=$ $\left\{g_{\alpha}^{\max \left\{j \mid g_{\alpha}^{j} \in \mathcal{G}\left(T^{j}\right), j<t\right\}}, x_{\beta}^{1}, \operatorname{FANIN}\left(s_{\gamma}\right)^{0}\right\}$. We must prove that $g_{i}^{t}$ in $T^{t}$ of $\mathbf{N}$ holds the value of gate $g_{i} @ t$ in $T$. In order to do so, we must show that the three types of fanins in $\operatorname{FANIN}\left(g_{i}^{t}\right)$ respectively hold the values of $g_{\alpha} @ t-1$, $x_{\beta} @ t-1$ and $s_{\gamma} @ t-1$.

1) Consider $g_{\alpha}^{\max }\left\{j \mid g_{\alpha}^{j} \in \mathcal{G}\left(T^{j}\right), j<t\right\}$. By the inductive hypothesis, time-gate $g_{\alpha}^{\max \left\{j \mid g_{\alpha}^{j} \in \mathcal{G}\left(T^{j}\right), j<t\right\}}$ holds the value of gate $g_{\alpha} @ \max \left\{j \mid g_{\alpha}^{j} \in \mathcal{G}\left(T^{j}\right), j<t\right\}$. Furthermore, by definition of $\mathcal{G}\left(T^{j}\right)$ in $(10)$ and $\mathcal{G}_{t}$, this is the last time-step before $t$ during which gate $g_{\alpha}$ can flip in the original circuit $T$. As such, timegate $g_{\alpha}^{\max \left\{j \mid g_{\alpha}^{j} \in \mathcal{G}\left(T^{j}\right), j<t\right\}}$ holds the value of gate $g_{\alpha} @ t-1$ in $T$.

2,3) Consider $x_{\beta}^{1}$ and $\operatorname{Fanin}\left(s_{\gamma}\right)^{0}$. Since $x_{\beta}\left(s_{\gamma}\right)$ is set to $x_{\beta}^{1}\left(s_{\gamma}^{1}=\operatorname{FANIN}\left(s_{\gamma}\right)^{0}\right)$ in $T$ at all time-steps $t \geq 0$, clearly $x_{\beta}^{1}\left(\operatorname{FANIN}\left(s_{\gamma}\right)^{0}\right)$ in $\mathbf{N}$ is equal to $x_{\beta} @ j\left(s_{\gamma} @ j\right)$ in $T, \forall j[0 \leq j<t]$, and in particular for $j=t-1$.

Since $g_{i}^{t}$ in $T^{t}$ of $\mathbf{N}$ performs the same logic function as $g_{i}$ in $T$ and its fanins hold the values of the fanins of $g_{i}$ at time $t-1$, it follows that $g_{i}^{t}$ holds the value of $g_{i} @ t$.

Lemma 1 shows that the values of the time-gates $g_{i}^{t}$ in $\mathbf{N}$ are consistent with the definition of $g_{i}^{t}\left(s^{0}, x^{0}, x^{1}\right)$ used in (9). As such, the final step is to add an XOR gate for every pair of $g_{i}^{t}$ and $g_{i}^{t^{\prime}}$, where there exists no $g_{i}^{t^{\prime \prime}}$ with $t<t^{\prime \prime}<t^{\prime}$ in $\mathrm{N}$. By construction, this is equivalent to adding an XOR gate between every $g_{i}^{t}$ and $g_{i}^{\max }\left\{j \mid g^{j} \in \mathcal{G}\left(T^{j}\right), j<t\right\}$. This is shown in Fig. 3. The weighted sum of these XOR gates yields:

$$
\sum_{t=1}^{\mathcal{L}} \sum_{g_{i} \in \mathcal{G}_{t}} C_{i} \cdot\left(g_{i}^{\max \left\{j \mid g^{j} \in \mathcal{G}\left(T^{j}\right), j<t\right\}}\left(s^{0}, x^{0}, x^{1}\right) \oplus g_{i}^{t}\left(s^{0}, x^{0}, x^{1}\right)\right)
$$

Moreover, since time-step $\max \left\{j \mid g^{j} \in \mathcal{G}\left(T^{j}\right), j<\right.$ $t\}$ is by definition the last time-step before $t$ in which gate $g_{i}$ could have flipped, it follows that the value of $g_{i}^{\max \left\{j \mid g^{j} \in \mathcal{G}\left(T^{j}\right), j<t\right\}}\left(s^{0}, x^{0}, x^{1}\right)$ is equal to that of $g_{i}^{t-1}\left(s^{0}, x^{0}, x^{1}\right)$. Replacing this in (11) yields (9), which is to be maximized by the PBO solver.

Example 3 Consider the circuit $T$ in Fig. 4 and the corresponding $\mathbf{N}$ in Fig. 3. Using a unit-delay model and not counting flips at DFF outputs or primary inputs, an optimal solution to the described $P B O$ problem is $\left\langle s^{0^{*}}, x^{0^{*}}, x^{1^{*}}\right\rangle=$ $\langle\langle 0\rangle,\langle 1,1,0\rangle,\langle 0,0,1\rangle\rangle$, which amounts to a total switched capacitance of 6 units as shown in Fig. 4.

The circuit $\mathbf{N}$ shown in Fig. 3 generates the activity produced by the triplet $\left\langle 0^{0^{*}}, x^{0^{*}}, x^{1^{*}}\right\rangle=\langle\langle 0\rangle,\langle 1,1,0\rangle,\langle 0,0,1\rangle\rangle$ as follows. Recall that $T^{t}(\forall t \geq 1)$ does not contain gates 


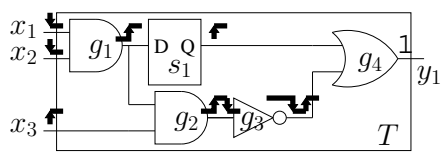

Fig. 4. Unit-delay gate switching in a sequential circuit

that cannot flip at time-step $t$, by construction. For instance, there is no time-gate corresponding to the inverter $g_{3}$ in $T^{1}$ because $g_{3}$ cannot flip at time-step 1 . Furthermore, as shown in Lemma 1, the interconnections in $\mathbf{N}$ are made such that each time-gate $g_{i}^{t}$ holds the value of $g_{i} @ t$ in $T$.

- In $T^{0}, g_{1}^{0}=1, g_{2}^{0}=0, g_{3}^{0}=1, g_{4}^{0}=1$. These values represent the initial state of the circuit (at time-step 0), before $x^{1 *}$ starts propagating inside.

- In $T^{1}, g_{1}^{1}=0, g_{2}^{1}=1, g_{4}^{1}=1$. Therefore, xor $_{1}=$ 1, xor $_{2}=1$, xor $_{6}=0$, yielding two gate flips and a total switched capacitance of 3 so far, since $C_{1}=2$.

- In $T^{2}, g_{2}^{2}=0, g_{3}^{2}=0, g_{4}^{2}=1$. Therefore, xor $_{3}=$ 1, xor $_{4}=1$, xor $_{7}=0$, yielding a total switched capacitance of 5 so far.

- In $T^{3}, g_{3}^{3}=1, g_{4}^{3}=1$. Therefore, xor $_{5}=1$, xor $_{8}=0$, yielding a total switched capacitance of 6 so far.

- Finally, in $T^{4}$, we have $g_{4}^{4}=1$. Therefore, $x_{0} r_{9}=0$ and the total switched capacitance is 6 .

Fig. 4 illustrates all gate switches on the original sequential circuit $T$.

The procedure outlined in this section can be extended to a more general delay model, where each gate has an arbitrary but fixed delay. This is done as follows. A linear time preprocessing step is described in [10], which generates, for each gate, the sequence of time instants at which it might flip. For each gate $g_{\alpha}$, let $t_{g_{\alpha}}^{i}$ and $t_{g_{\alpha}}^{f}$ respectively denote the first and last time instants at which $g_{\alpha}$ might flip. A circuitlevel time sequence that includes all possible gate flipping time instants can be subsequently created. In order to apply the methodology described in this section to an arbitrary delay model, for each gate $g_{\alpha}, l\left(g_{\alpha}\right)$ and $L\left(g_{\alpha}\right)$ should be respectively set to the indices of $t_{g_{\alpha}}^{i}$ and $t_{g_{\alpha}}^{f}$ in the sorted circuit-level time sequence. Note that this generalization is not applicable if the delay of each gate is variable or is given by an interval of possible values.

It should be noted that using a general delay model would significantly increase the size of the circuit $\mathbf{N}$ because the number of time instants at which the inputs of a given gate can switch scales exponentially with the topological level of the gate (i.e., its min-level) [16]. This is in contrast to unitdelay, where the number of time instants at which the inputs of a given gate can switch scales linearly with its min-level. The authors of [16] propose a viable approach to allow such techniques to handle general gate delays as well as larger circuits, by splitting the problem into smaller subproblems that are easier to solve, using a sequence of spatial and temporal windows. Furthermore, advances in PBO solvers will increase the applicability of our method.

\section{INPUT CONSTRAINTS}

Digital designs might operate under certain assumptions, where some input patterns are considered illegal or unlikely to occur. Not taking these assumptions into account can produce unrealistic maximum activity estimates which can result in an over-conservative design. In this section, we outline how to add input or state constraints to the problem to exclude unwanted input combinations or sequences of input combinations. For instance, the following is an example of such a constraint. Given the initial state $s^{0}=\langle 0,0, X, X\rangle$, the input sequence $\left\langle x^{0}, x^{1}\right\rangle=\langle\langle X, 1,0\rangle,\langle 1,0, X\rangle\rangle$ is illegal. Here $X$ denotes a don't-care. This constraint can be translated to the following SAT clause:

$$
\left(s_{1}^{0} \vee s_{2}^{0} \vee \bar{x}_{2}^{0} \vee x_{3}^{0} \vee \bar{x}_{1}^{1} \vee x_{2}^{1}\right)
$$

During the PBO search, any assignment of the triplet $\left\langle s^{0}, x^{0}, x^{1}\right\rangle$ that violates the given condition will produce a conflict due to this clause, forcing the solver to backtrack from unwanted parts of the search-tree.

Similarly, sets of unreachable initial-state cubes, possibly including don't-cares, can be ruled out from the search. For instance, the illegal initial-state cubes $s^{0}=\langle 1, X, 0\rangle$ and $s^{0}=$ $\langle 1,1,1\rangle$ can be ruled out with the two SAT clauses: $\left(\bar{s}_{1}^{0} \vee\right.$ $\left.s_{3}^{0}\right) \wedge\left(\bar{s}_{1}^{0} \vee \bar{s}_{2}^{0} \vee \bar{s}_{3}^{0}\right)$. The interested reader can refer to [34] for a discussion on sequential reachability, which is outside the scope of this work.

Furthermore, it is also possible to rule out input sequences that are deemed as unlikely. Here we consider a type of constraint limiting the number of bit flips in the primary inputs to at most $d$ flips. In other terms, the Hamming distance between $x^{0}$ and $x^{1}$ is constrained to be less than or equal to $d$ :

$$
\sum_{i} x_{i}^{0} \oplus x_{i}^{1} \leq d
$$

In this case, a naive encoding into clauses that does not use auxiliary variables will blow up in memory, especially if $d$ is large. In order to express this type of constraints using clauses, first an XOR gate $a_{i}=x_{i}^{0} \oplus x_{i}^{1}$ is added in $\mathbf{N}$ for every primary input bit $x_{i}$ in $T$. Next, we construct a bitonic sorter [29] using AND and OR gates, which takes in the Boolean variables $a_{1}, a_{2}, \ldots$ and generates the corresponding sorted output sequence in decreasing order, denoted as $b_{1}, b_{2}, \ldots$. Finally, the unit clause $\left(\bar{b}_{d+1}\right)$ is added to the problem, forcing $b_{d+1}=0$. Since the $b_{i}$ 's are sorted in decreasing order, this will automatically force $b_{d+2}=0, b_{d+3}=0, \ldots$. Consequently at most $d$ bits in the output of the bitonic sorter $\left(b_{1}, \ldots, b_{d}\right)$ can be set to 1 by the solver. Hence, by construction, at most $d$ bits in $a_{1}, a_{2}, \ldots$ can be set to 1 , which means that at most $d$ primary inputs can flip simultaneously. This construction requires $O\left(|x| \log ^{2}|x|\right)$ clauses, where $|x|$ denotes the number of primary inputs [22].

\section{OptimizATIONS AND HEURISTICS}

In this section, optimization techniques are presented for improving the PBO formulation. 


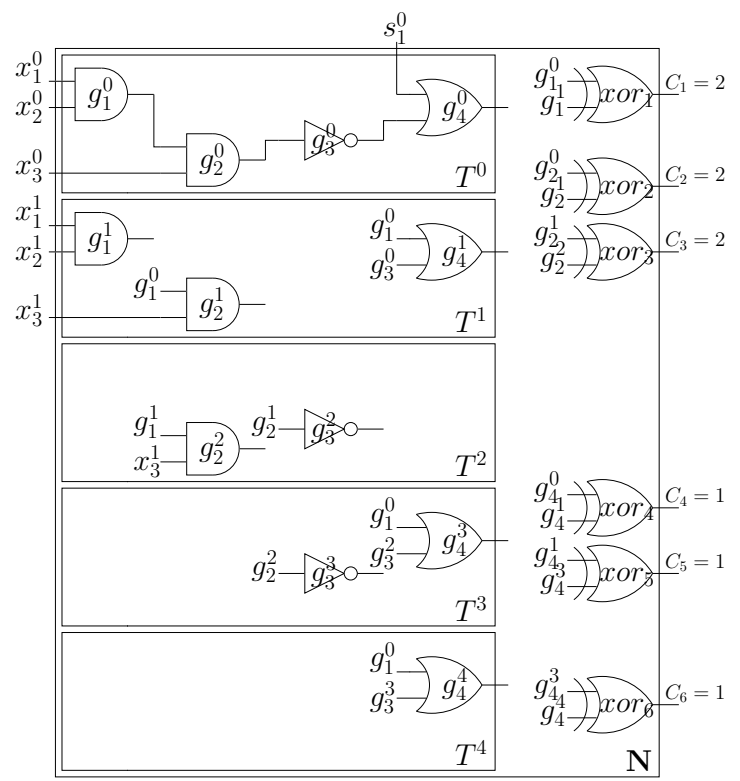

Fig. 5. Optimized PBO formulation, unit-delay model

\section{A. Reduction of $\mathcal{G}_{t}$}

The definition of $\mathcal{G}_{t}$ given in Definition 3 can be tightened. In fact, irrespective of the triplet $\left\langle s^{0}, x^{0}, x^{1}\right\rangle$, it is sometimes known in advance that a certain gate $g_{i}$ can never flip at timestep $t$ even though $g_{i} \in \mathcal{G}_{t}$. This can happen if $l\left(g_{i}\right) \leq t \leq$ $L\left(g_{i}\right)$, but there exists no path $p$ of length exactly $t(|p|=t)$ from a primary input or DFF output to the output of $g_{i}$. For example, in the circuit of Fig. 2(a), although $l\left(g_{4}\right)=1$ and $L\left(g_{4}\right)=4, g_{4}$ can never flip at time-step 2. Hence, in Fig. 3, the time-gate $g_{4}^{2}$ is redundant because its output will always be the same as that of $g_{4}^{1}$. The following is a tighter definition of $\mathcal{G}_{t}$.

Definition $4 \mathcal{G}_{t}=\left\{g_{i} \in \mathcal{G}(T) \mid \exists\right.$ a path $p$ from a primary input or state to $g_{i}$ with $\left.|p|=t\right\}$

In other terms, $\mathcal{G}_{t}$ is the set of all gates reachable in exactly $t$ steps from a primary input or a DFF output. The sets $\mathcal{G}_{1}, \mathcal{G}_{2}, \ldots, \mathcal{G}_{\mathcal{L}}$ can be generated using a variation of breadthfirst traversal of the original circuit, starting from primary inputs and pseudo-inputs and memorizing the set of newly reached gates at each time-step. In Fig. 5, $\mathbf{N}$ is optimized to use Definition 4 for $\mathcal{G}_{t}$.

\section{B. Sequences of BUFFERs and/or NOTs}

Suppose gate $g_{i}$ is a BUFFER or a NOT. If the input of $g_{i}$ flips, then the output of $g_{i}$ flips. Therefore, for every sequence of BUFFERs and/or NOTs, it is sufficient to put only one XOR at the input of the first BUFFER/NOT and to add the load capacitances of the other gates to the weight of this XOR. In Fig. 5, this optimization is used to reduce the number of XORs. For large circuits with significant numbers of NOTs and BUFFERs, this can significantly reduce the size of the $\mathbf{N}$ as well as $\mathcal{F}$, and therefore the number of pseudo-Boolean constraints.

\section{Non-zero Initial Activity using Simulations}

As described in Subsection III-B, the PBO solver gradually tightens the upper bound on the objective function, and therefore the lower bound on maximum circuit activity. This is done until either the maximum is proved or the solver timesout. However, instead of starting from an activity of 0 , it is possible to first run random simulations for $R$ seconds, record the generated maximum activity $M$ and then force the solver to start from an activity of at least $\alpha \cdot M$, for some user-specified $\alpha \in[0,1]$, using an appropriate pseudoBoolean constraint. If $\alpha$ is close to 1, this has the advantage of guiding the solver into parts of the search-space that might potentially yield higher circuit activities, and saves it the time of finding possibly many suboptimal solutions in other parts of the search-space. However, this will make the initial PBS problem harder. Therefore finding the first solution that yields a circuit activity greater than $\alpha \cdot M$ may take a longer time. Moreover, the PBO solver may have a harder time learning from its mistakes.

\section{Gate Switching Equivalence Classes}

Subsection VIII-B considers a special case of several gates always switching together for sequences of BUFFER and/or NOT gates. In fact, this may happen in more general cases. Also, due to structural correlations, some gates are more likely to switch in tandem. We utilize such gate switching correlations by building equivalence classes for simultaneously switching gates as follows.

We run random simulations for $R$ seconds. Each gate or time-gate is associated with its switching signature, which is a sequence of $0 \mathrm{~s}$ and $1 \mathrm{~s}$ indicating switching times based on these simulations. For each gate, a 1 indicates a switch, while a 0 indicates that no switch occurred for a given input vector. In the case of non-zero gate delays, the switching of each timegate is recorded (this is equivalent to recording all glitches of a gate during the simulation of the original circuit).

Next, these gates are sorted in their signatures' lexicographical order (i.e., in increasing order of the binary numbers given by these signatures. For example, $001010<010001$ ). Successive gates (or time-gates) with equal signatures are grouped into the same equivalence class. These gates (or time-gates) are assumed to be likely to switch simultaneously, although they are not guaranteed to do so.

In the construction of $\mathbf{N}$, the "switch detecting" XOR gates described in this work are only added for one representative gate (or time-gate) in each equivalence class. Furthermore, all the output capacitances of the gates in an equivalence class are now added to the weight of the XOR of the representative in the objective function. Less added XORs leads to a smaller $\mathcal{F}$, which results in a smaller PBO to SAT translation in the solver [22], improving the scalability of the technique.

The downside is that the resulting circuit activities returned by the PBO solver can now contain a small error, due to the fact that gates belonging to the same equivalence class might not always switch together. In order to avoid returning "false positive" (i.e., unrealizable) switching activities due to this approximation, we always simulate the resulting solutions 


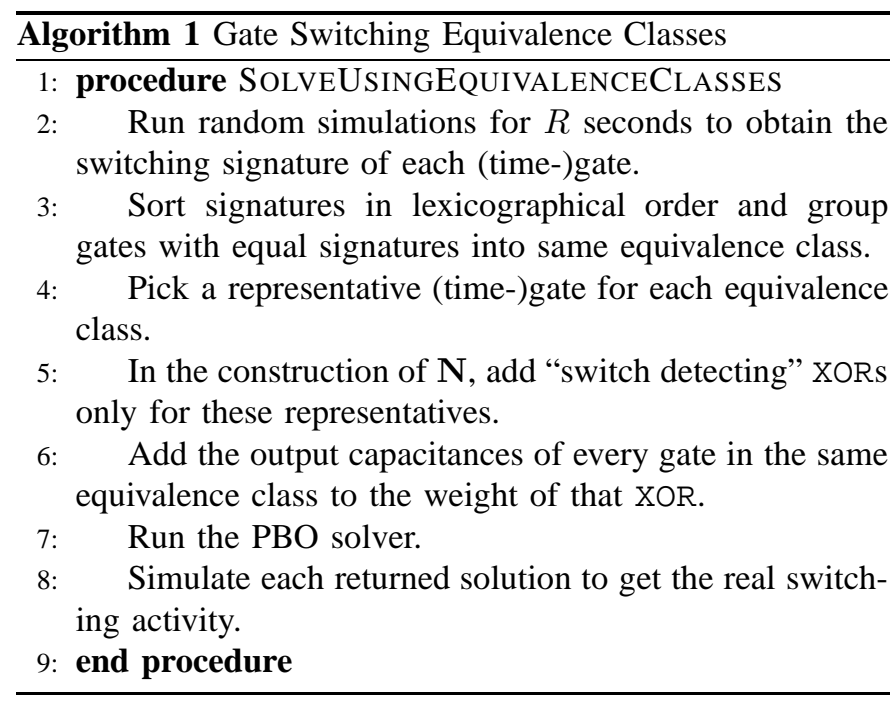

(input sequences) returned by the solver and record their real switching activities. Here, we can generate a trade-off based on the original simulation time $R$ to set up the equivalence classes. The longer $R$, the more accurate the results of the PBO solver will be, but the smaller the size of gate switching equivalence classes and therefore the bigger the problem size. With shorter simulation times, the formulation will be smaller, however there will be more noise in the solution returned by the PBO solver. It should be emphasized that this approximation can miss input transitions that cause more switching in the original circuit and therefore cannot be used to prove that the maximum circuit activity has been found.

\section{EXPERIMENTAL RESUlTS}

The zero-delay and unit-delay formulations of the proposed PBO-based approach for circuit activity estimation are implemented in $\mathrm{C}++$. The resulting $\mathrm{PBO}$ problem is solved using the MiniSAT+ [22] engine. The optimizations given in Subsections VIII-A and VIII-B are integrated into the formulation by default, and experiments are run with and without the heuristics described in Subsections VIII-C and VIII-D. All experiments are conducted on a Pentium IV $2.8 \mathrm{GHz}$ Linux platform with 2 GB of memory.

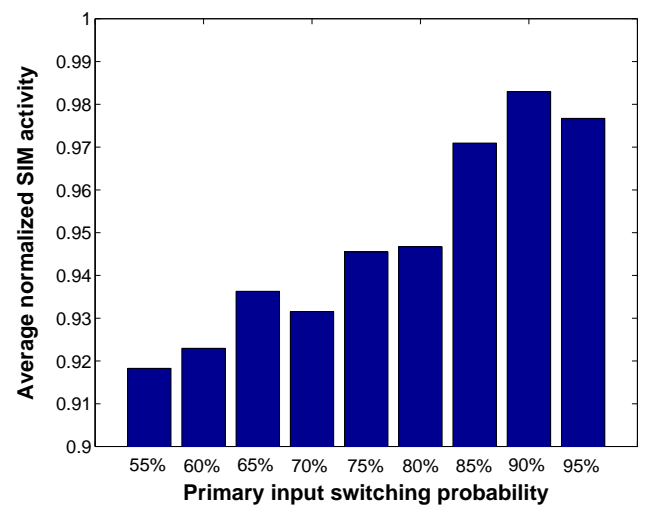

Fig. 6. Normalized SIM activity versus $p$ given 100 seconds
Our approach is compared to parallel-pattern random simulations, referred to as SIM, with 32-bit words (32 simultaneous vector simulations). In SIM, let $p$ denote the (user-specified) probability that a given primary input flips. In other terms, $\forall i$, let $\operatorname{Pr}\left(x_{i}^{0} \neq x_{i}^{1}\right)=p$. We have experimented with several values of $p$ ranging from $55 \%$ to $95 \%$, using a time-out of 100 seconds and a representative set of thirty instances from ISCAS85 and ISCAS89 circuits, using both zero and unitdelay models. Fig. 6 shows the results in the form of average normalized SIM activities for each input switching probability $p$. More precisely, for each instance and each primary input switching probability $p$, we compute the ratio of the generated activity to the maximum activity for that instance among all switching probabilities. It can be seen that $p=90 \%$ yields the highest average normalized activity (0.983), whereas $95 \%$ and $85 \%$ respectively yield ratios of 0.977 and 0.971 . Lower $p$ 's yield inferior activities, with $p=55 \%$ resulting in the lowest average ratio of 0.918 . Therefore, the switching probability of each primary input in SIM in the remainder of our experiments is set to $90 \%$. This value is also consistent with [9].

For sequential circuits, SIM continuously picks a new, arbitrary, initial state $s^{0}$, and applies $x^{0}, x^{1}$ from $s^{0}$. This guarantees a fair comparison with the PBO-based approach, which can also explore arbitrary initial states. At the end of this section, we provide experimental results using input constraints for both of these methods. The time-out is set to 10000 seconds, and the generated sequence of increasing switching activities along with their corresponding run-times is recorded for each experiment. Depending on the size of the circuit, roughly a million to forty million vectors are simulated in 10000 seconds for SIM.

On the other hand, three sets of PBO experiments are performed. The first is the original formulation for combinational or sequential circuits, with zero or unit-delay. This includes the $\mathcal{G}_{t}$ reduction technique described in Subsection VIII-A, as well as the optimization for BUFFER/NOT sequences given in Subsection VIII-B. The second set of experiments adds to this the heuristic given in Subsection VIII-C. Here $R=5$ seconds of random simulations are run to find an initial maximum activity $M$ before our method is applied, and the PBO solver is forced to start from an activity of at least $\alpha \cdot M$ with $\alpha=0.9$. This value is chosen as a compromise between an $\alpha$ which yields an original problem that is too difficult to solve (e.g. $\alpha \geq 1$ ) and one that has little effect on the solver. The third set of experiments adds the switching equivalence classes heuristic described in Subsection VIII-D to the original PBO formulation. Here $R=2$ seconds of random simulations are performed to obtain the switching signatures of each gate or time-gate.

Tables I and II show the experimental results for ten ISCAS85 and twenty ISCAS89 benchmarks, respectively. We describe these two tables simultaneously because they have the same structure. The first and second rows respectively show the circuit names and the corresponding numbers of gates. The maximum circuit activities in Tables I and II are in units of switched capacitance, where $C_{i}=\mid$ FAnOUTS $\left(g_{i}\right) \mid$ for internal gates and $C_{i}=1$ for primary output gates. For each experiment, the generated maximum activity values are 
TABLE I

MAXIMUM ACTIVITIES PER CYCLE OBTAINED BY PBO AND SIM FOR COMBINATIONAL CIRCUITS

\begin{tabular}{|c|c|c|c|c|c|c|c|c|c|c|c|c|c|}
\hline & $\bar{T}$ & c432 & c499 & c880 & c1355 & c1908 & c2670 & c3540 & $\mathbf{c 5 3 1 5}$ & c6288 & c7552 \\
\hline & & & $|\mathcal{G}(T)|$ & 164 & 555 & 381 & 549 & 404 & 709 & 965 & 1579 & 3398 & 2325 \\
\hline \multirow{12}{*}{$\begin{array}{l}\text { zero } \\
\text { delay }\end{array}$} & \multirow{9}{*}{ PBO } & & $100 \mathrm{~s}$ & ${ }^{*} 193$ & 441 & 470 & $\overline{447}$ & 430 & $\overline{753}$ & $\overline{1053}$ & 1395 & 3023 & 2064 \\
\hline & & & $1000 \mathrm{~s}$ & *193 & 493 & 482 & 480 & 445 & 754 & 1054 & 1611 & 3191 & 2282 \\
\hline & & & $10000 \mathrm{~s}$ & *193 & 493 & ${ }^{*} 482$ & 480 & 456 & 773 & 1058 & 1689 & 3678 & 2544 \\
\hline & & \multirow{3}{*}{$\begin{array}{c}+ \text { VIII-C } \\
R=5 s \\
\alpha=0.9\end{array}$} & $100 \mathrm{~s}$ & ${ }^{*} 193$ & 462 & 476 & 462 & 433 & 744 & 979 & 1578 & 3078 & \\
\hline & & & $1000 \mathrm{~s}$ & ${ }^{*} 193$ & 471 & 481 & 471 & 441 & 764 & 1008 & 1593 & 3497 & 2236 \\
\hline & & & $10000 \mathrm{~s}$ & ${ }^{*} 193$ & 485 & ${ }^{*} 482$ & 479 & 459 & 775 & 1032 & 1638 & 3497 & 2620 \\
\hline & & \multirow{3}{*}{$\begin{array}{c}+ \text { +VIII-D } \\
R=2 s\end{array}$} & $100 \mathrm{~s}$ & 193 & 478 & 474 & 457 & 427 & 727 & 973 & 1528 & 3449 & 2135 \\
\hline & & & $1000 \mathrm{~s}$ & 193 & 482 & 482 & 473 & 443 & 739 & 1041 & 1606 & 3449 & 2399 \\
\hline & & & $10000 \mathrm{~s}$ & 193 & 485 & 482 & 480 & 459 & 773 & 1053 & 1654 & 3449 & 2484 \\
\hline & \multirow{3}{*}{ SIM } & & $100 \mathrm{~s}$ & 176 & 421 & 414 & 424 & 389 & 657 & 938 & 1470 & 2980 & 2179 \\
\hline & & & $1000 \mathrm{~s}$ & 178 & 421 & 437 & 426 & 389 & 659 & 938 & 1470 & 3107 & 2198 \\
\hline & & & $10000 \mathrm{~s}$ & 178 & 444 & 437 & 426 & 389 & 671 & 950 & 1476 & 3195 & 2222 \\
\hline \multirow{12}{*}{$\begin{array}{l}\text { unit } \\
\text { delay }\end{array}$} & \multirow{9}{*}{ PBO } & & $100 \mathrm{~s}$ & 838 & 2172 & 1330 & 2647 & 2133 & 2082 & 2633 & 7140 & 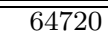 & 6198 \\
\hline & & & $1000 \mathrm{~s}$ & 1006 & 2713 & 2508 & 2770 & 2176 & 2467 & 5096 & 7140 & 64720 & 6198 \\
\hline & & & $10000 \mathrm{~s}$ & ${ }^{*} 1041$ & 2779 & 2743 & 2781 & 2720 & 2779 & 6670 & 8034 & 64720 & 10477 \\
\hline & & \multirow{3}{*}{$\begin{array}{l}+ \text { VIII-C } \\
R=5 s \\
\alpha=0.9\end{array}$} & $100 \mathrm{~s}$ & 879 & 2494 & 1712 & & 2691 & 2466 & & & & \\
\hline & & & $1000 \mathrm{~s}$ & 941 & 2741 & 3103 & 2974 & 2720 & 2605 & & & & \\
\hline & & & $10000 \mathrm{~s}$ & ${ }^{*} 1041$ & 2900 & 3196 & 2987 & 3329 & 2804 & 6813 & 8706 & 101921 & 12744 \\
\hline & & \multirow{3}{*}{$\begin{array}{c}\text { +VIII-D } \\
R=2 s\end{array}$} & $100 \mathrm{~s}$ & 902 & 2277 & 3056 & 2487 & 2510 & 2503 & 3809 & 3895 & 82055 & 6195 \\
\hline & & & $1000 \mathrm{~s}$ & 1032 & 2928 & 3056 & 2562 & 2524 & 2725 & 4776 & 7178 & 96622 & 6195 \\
\hline & & & $10000 \mathrm{~s}$ & 1041 & 2928 & 3056 & 2909 & 2896 & 2886 & 6332 & 8583 & 147010 & 13517 \\
\hline & \multirow{3}{*}{ SIM } & & $100 \mathrm{~s}$ & 880 & 2292 & 2366 & 2234 & 2500 & 2508 & 6540 & 8100 & 118364 & 11699 \\
\hline & & & $1000 \mathrm{~s}$ & 949 & 2299 & 2366 & 2348 & 2509 & 2605 & 6570 & 8199 & 129672 & 11811 \\
\hline & & & $10000 \mathrm{~s}$ & 964 & 2316 & 2366 & 2450 & 2509 & 2606 & 6596 & 8216 & 139341 & 11900 \\
\hline
\end{tabular}

recorded after 100, 1000 and 10000 seconds. For each circuit and delay model, activities are compared between the four sets of experiments, namely, PBO, PBO+VIII-C with $R=5$ seconds and $\alpha=0.9$, PBO+VIII-D with $R=2$ seconds and finally SIM. The highest activity after each time-period is highlighted in bold. An empty table cell indicates that no bound is found up to that time. Finally, a " $*$ " in front of an activity value indicates that the $\mathrm{PBO}$ solver proved that the generated activity is in fact the absolute maximum. This occurs if the incremental PBS formula becomes UNSAT, signaling that no higher circuit activity can be found.

For instance, using a unit-delay model, in circuit c432, both $\mathrm{PBO}$ and $\mathrm{PBO}+\mathrm{VIII}-\mathrm{C}$ prove the maximality of 1041 by 10000 seconds, whereas the maximum activity generated by SIM is (964). Note that for PBO+VIII-D, the activities found by the solver are not recorded directly since they are not guaranteed to be exact due to uncertainties in switching equivalence classes, as described in Subsection VIII-D. Instead, the corresponding input sequences returned by the solver

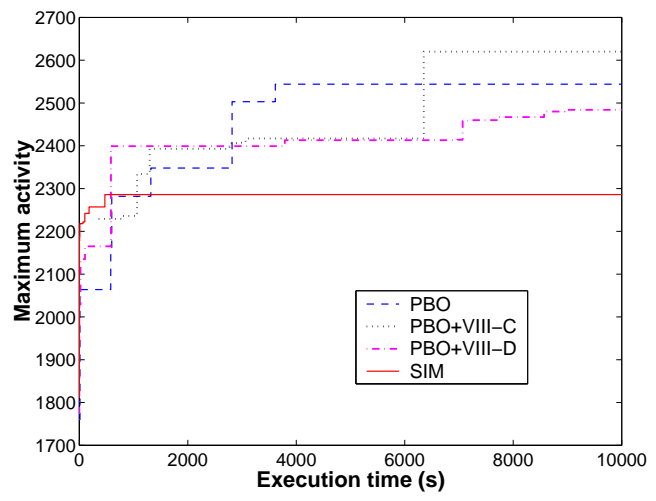

Fig. 7. Maximum activity versus execution time for c7552, zero-delay model are simulated on the circuit and the resulting activities are recorded. As a result, even when PBO+VIII-D "proves" the maximality of a switching activity, this is not shown using a "*" in Tables I and II.

Overall, by the 10000 second time-out, PBO, PBO+VIII-C and PBO+VIII-D respectively yield $6 \%, 7 \%$ and $7 \%$ higher activities than SIM on average. Our approaches yield an average $11 \%$ improvement over simulations using a zero-delay model, and 3\% improvement using a unit-delay model. All these averages are pushed down by some of the negative results of the PBO-based approaches for the largest circuits, such as s38584. At the 100 and 1000 second marks, SIM slightly outperforms the PBO-based approaches. However, a longer timeout benefits our methods. There exists previous work [9] that provides a statistical quality measure for maximum activities generated using simulations. On the other hand, attempting to estimate a reasonable PBO time-out is a very difficult endeavor because of the nature of SAT and PBO solvers, in the sense that it is difficult to predict their performance. The assumption

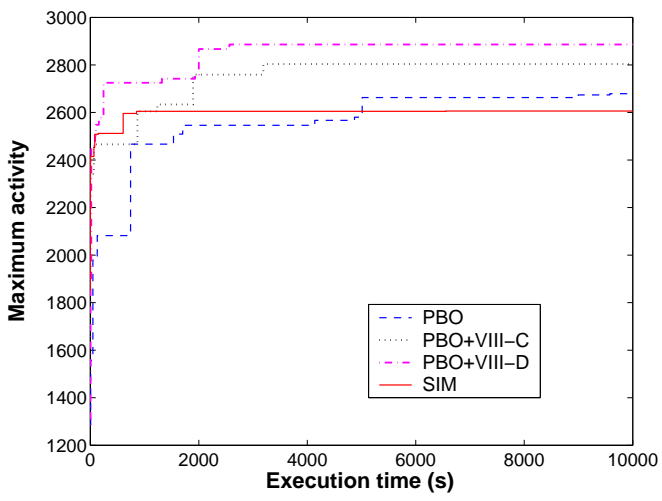

Fig. 8. Maximum activity versus execution time for c2670, unit-delay model 
TABLE II

MAXIMUM ACTIVITIES PER CYCLE OBTAINED BY PBO AND SIM FOR SEQUENTIAL CIRCUITS

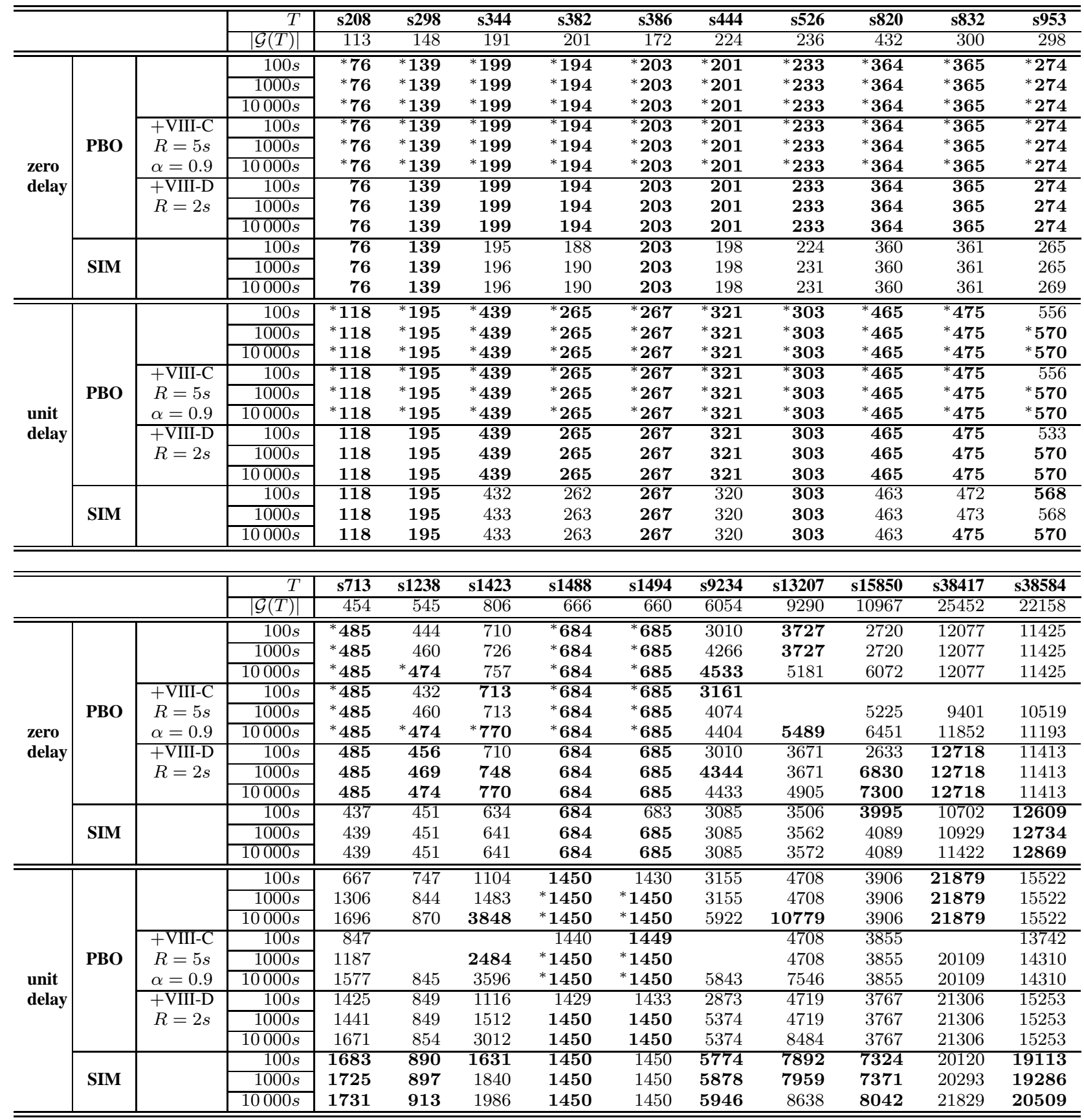

is that these power estimation runs can be performed by the engineer overnight and therefore can have reasonably long time-outs, which benefits our method.

Fig. 7 and Fig. 8 show the circuit activities generated by each of the methods for c7552 with zero-delay and c2670 with unit-delay, plotted against execution time. A common observation in these figures, as well as in Tables I and II, is that SIM results tend to plateau, whereas PBO-based approaches continue producing increasing activities. However, in some cases where the size of the symbolic problem starts affecting the performance of the PBO-based approach (e.g. for benchmark s15850), even 10000 seconds is not enough time for MINISAT+ to improve the maximum activity significantly.
There exist cases where the estimation improvement of PBO-based approaches compared to SIM is considerably large. For instance, using a unit-delay model, in circuit s1423, PBO, PBO+VIII-C and PBO+VIII-D respectively record 94\%, $81 \%$ and $52 \%$ improvements over SIM. This supports the argument that our PBO-based technique, being an exhaustive symbolic approach, complements simulations by occasionally discovering "hidden" corner cases of maximum activity generating stimuli that are missed by SIM.

Benchmark c6288 with unit-delay constitutes a special case because of its disproportionately large number of levels $(\mathcal{L}=$ 164 ), which causes $\mathbf{N}$, and subsequently the CNF of the SAT problem, to be very large. As a result, for c6288 with unit- 
TABLE III

SWITCHING EQUIVALENCE CLASSES

\begin{tabular}{|c|c|c|c|c|c|c|c|c|c|c|c|}
\hline & $T$ & $\mathbf{c 4 3 2}$ & c499 & c880 & c1355 & $\mathbf{c 1 9 0 8}$ & $\overline{c 2670}$ & $\overline{c 3540}$ & $\mathbf{c 5 3 1 5}$ & c6288 & $\overline{\mathbf{c 7 5 5 2}}$ \\
\hline \multirow{2}{*}{$\begin{array}{l}\text { zero } \\
\text { delay }\end{array}$} & \# switch XORs & $\overline{129}$ & 421 & 302 & $\overline{420}$ & $\overline{312}$ & $\overline{576}$ & 785 & 1305 & 2363 & 1774 \\
\hline & \# equivalence classes $(R=2 s)$ & 129 & 412 & 299 & 413 & 310 & 542 & 730 & 1273 & 2359 & 1679 \\
\hline \multirow{3}{*}{$\begin{array}{l}\text { unit } \\
\text { delay } \\
\end{array}$} & \# switch XORs & 1053 & 3565 & 3061 & 3669 & 4011 & 3199 & 9666 & 11132 & 143422 & 19428 \\
\hline & \# equivalence classes $(R=2 s)$ & 1041 & 3284 & 2733 & 3316 & 3522 & 2616 & 8066 & 9437 & 94638 & 15899 \\
\hline & ( & s713 & $\mathbf{s 1 2 3 8}$ & s1423 & s1488 & s1494 & 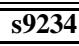 & $\mathbf{s 1 3 2 0 7}$ & $\mathbf{s 1 5 8 5 0}$ & $\mathbf{\text { s38417 }}$ & $\mathbf{s 3 8 5 8 4}$ \\
\hline \multirow{2}{*}{$\begin{array}{l}\begin{array}{l}\text { zero } \\
\text { delay }\end{array} \\
\end{array}$} & \# switch XORs & $\overline{168}$ & 451 & $\overline{526}$ & $\overline{566}$ & 571 & $\overline{2194}$ & 3048 & 3766 & 10345 & $\overline{12794}$ \\
\hline & \# equivalence classes $(R=2 s)$ & 131 & 446 & 481 & 537 & 544 & 1720 & 2303 & 2985 & 9109 & 11254 \\
\hline \multirow{2}{*}{$\begin{array}{l}\text { unit } \\
\text { delay }\end{array}$} & \# switch XORs & 3198 & 2497 & 10186 & 2194 & 2213 & 17241 & 14126 & 42765 & 61748 & 75973 \\
\hline & \# equivalence classes $(R=2 s)$ & 1093 & 1998 & 2293 & 1871 & 1887 & 5415 & 4330 & 6758 & 28364 & 26994 \\
\hline
\end{tabular}

delay, we explicitly tell MiniSAT+ to use '-adders' (see [22]) in order to save memory, at the expense of performance.

Fig. 9, Fig. 10 and Fig. 11 plot the activities generated by $\mathrm{PBO}, \mathrm{PBO}+\mathrm{VIII}-\mathrm{C}$ and PBO+VIII-D, respectively, against those generated by SIM, on a logarithmic scale. These numbers are compared after 100, 1000 and 10000 seconds. In all cases, it can be seen that longer time-outs help PBO over SIM, given that simulation results start to plateau. In Fig. 9, after 100 and 1000 seconds, many points are still below the $45^{\circ}$ line. However, after 10000 seconds, the PBO activities mostly beat SIM activities with some exceptions. For PBO+VIII-C, Fig. 10 and Table I only record the activities found by the PBO solver and do not show the activities generated by the first $R=5$ seconds of simulations. As expected, the PBO solver sometimes requires more than 100 seconds to find circuit activities exceeding the maximum found by simulations after $R=5$ seconds. The 10000 second points in Fig. 10 are generally above the $45^{\circ}$ line. Finally, in Fig. 11, the use of equivalence classes improves the scalability of the larger problems. In particular, the activity found using PBO+VIII-D for c6288 with unit-delay is the only one that surpasses that found by SIM within 10000 seconds.

Table III shows the number of switching equivalence classes found using $R=2$ seconds of simulation (and hence the number of "switch" XOR gates in $\mathbf{N}$ ) for the ten largest ISCAS89 circuits and all ISCAS85 circuits. This number is compared to the original number of XOR gates in $\mathbf{N}$ without the heuristic of Section VIII-D. One can notice that the reduction in added XORs increases with the circuit size. This is expected, since given a constant simulation time, less vectors can be simulated for a large circuit compared to a small design,

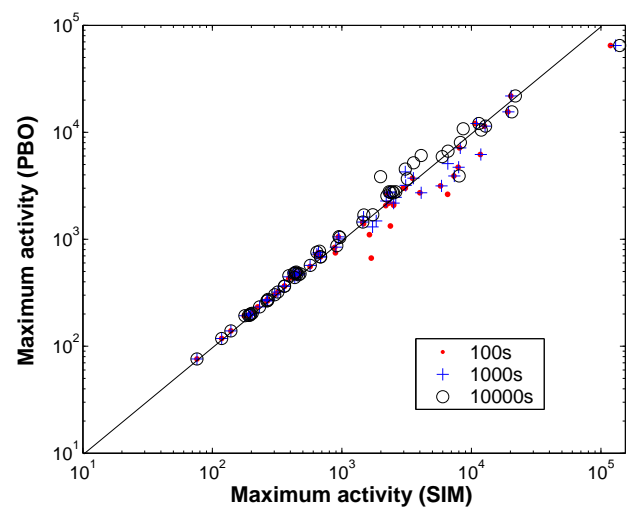

Fig. 9. SIM vs. PBO maximum activities

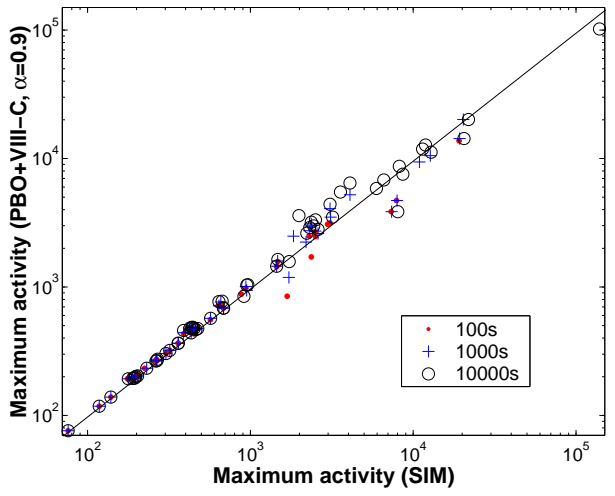

Fig. 10. SIM vs. PBO+VIII-C maximum activities

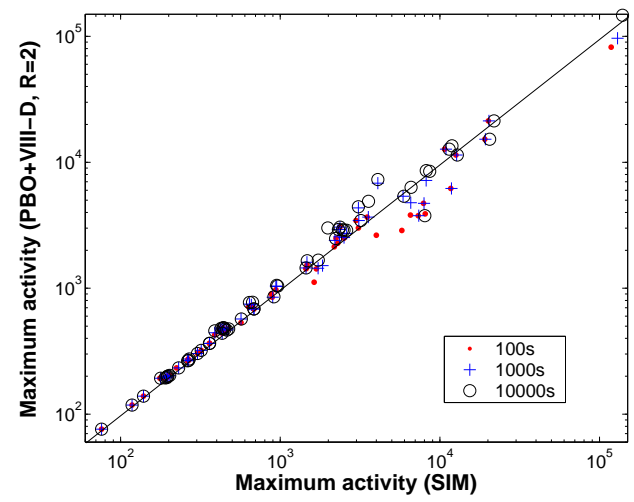

Fig. 11. SIM vs. PBO+VIII-D maximum activities

and therefore gates or time-gates will be less differentiated during simulations, resulting in larger equivalence classes. The argument for doing this is that a reduction is more useful for larger problems in order to extend scalability. The advantage of using switching equivalences classes becomes especially apparent for the c6288 benchmark with unit-delay, where due to the prohibitively large $\mathcal{F}$ in the original formulation, MINISAT + is unable to improve on its original activity estimates using PBO (64720) and PBO+VIII-C (101921). On the other hand, using PBO+VIII-D, the found maximum activity is improved several times.

In order to observe the effects of a longer time-out, we pick ten circuits, where, using a unit-delay model, SIM either outperforms PBO or yields only slightly lower activities within 10000 seconds, as shown in Tables I and II. We increase the time-out to 50000 seconds, and record the generated activities in Table IV, next to the 10000 second time-out results. Whereas in only 2 of these circuits (s13207 and 


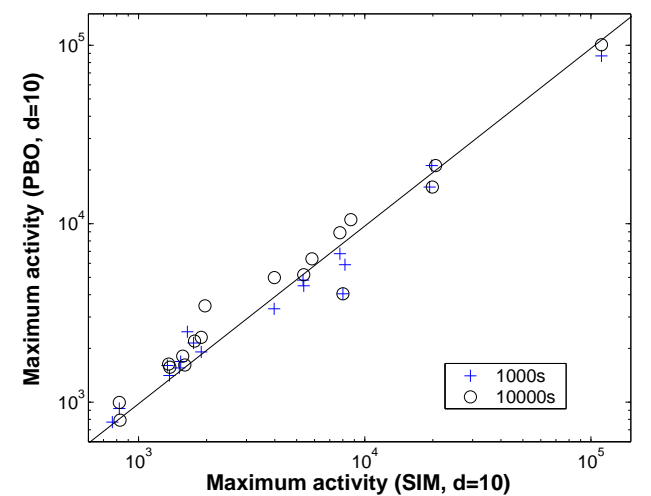

Fig. 12. SIM vs. PBO with at most $d=10$ input flips, unit-delay model

s38584) PBO outperforms SIM within 10000 seconds, this number increases to 7 within 50000 seconds. Overall, PBO activities increase by $30 \%$ on average from the 10000 to the 50000 second mark, whereas SIM activities increase by a mere $1 \%$. This is not surprising because MINISAT+ keeps learning clauses and focusing its search in an attempt to exhaust the problem search-space, whereas SIM continues to blindly apply pseudo-random simulations. Furthermore, PBO is able to find the maximum circuit activity for s713 within 50000 seconds.

However, this indicates that the PBO activities generated within 10000 seconds for the circuits in Table IV are on average at least 30\% lower than their actual maximum activities. As such, it is useful to investigate the time required by the PBO solver to arrive at reasonably accurate maximum activity estimates. Unfortunately, there is no clear relationship between circuit size and this required time-out, due to the unpredictable behavior of PBO solvers. Furthermore, the incremental improvements in activities returned by the PBO solver do not necessarily become smaller with time. As such, it is not possible to use the sequence of returned PBO activities in a vacuum to determine a time-out. On the other hand, some $\mathrm{PBO}$ solvers, e.g. MINIS AT+, regularly report a progress value at run-time, measuring the percentage of the searchspace that has been visited or pruned so far. This could be used in determining when to stop the solver. A more robust option would be to use a statistical method such as $[6,14]$ as a preliminary maximum activity estimation step, which is to be confirmed by an actual input pattern returned by PBO. In this case, the PBO solver would be stopped if an activity close to the statistical estimation has been found, or if some

TABLE IV

PBO VERSUS SIM RESULTS WITH A 50000 SECONDS TIME-OUT

\begin{tabular}{l|r|r|r|r}
\hline \multirow{2}{*}{$T$} & \multicolumn{2}{|c|}{ PBO } & \multicolumn{2}{c}{ SIM } \\
\cline { 2 - 5 } & $10000 s$ & \multicolumn{1}{|c}{$50000 s$} & $10000 s$ & \multicolumn{1}{c}{$50000 s$} \\
\hline \hline $\mathbf{c 5 3 1 5}$ & 8034 & $\mathbf{8 6 3 3}$ & $\mathbf{8 2 1 6}$ & 8427 \\
\hline $\mathbf{c 6 2 8 8}$ & 64720 & 111346 & $\mathbf{1 3 9 3 4 1}$ & $\mathbf{1 4 2 3 7 5}$ \\
\hline $\mathbf{c 7 5 5 2}$ & 10477 & 12569 & $\mathbf{1 1 9 0 0}$ & $\mathbf{1 2 7 1 3}$ \\
\hline \hline $\mathbf{s 7 1 3}$ & 1696 & ${ }^{*} \mathbf{1 8 2 9}$ & 1731 & 1739 \\
\hline $\mathbf{s 1 2 3 8}$ & 870 & 884 & $\mathbf{9 1 3}$ & $\mathbf{9 1 3}$ \\
\hline $\mathbf{s 9 2 3 4}$ & 5922 & $\mathbf{6 1 5 7}$ & $\mathbf{5 9 4 6}$ & 6005 \\
\hline $\mathbf{s 1 3 2 0 7}$ & $\mathbf{1 0 7 7 9}$ & $\mathbf{1 1 7 4 0}$ & 8638 & 8638 \\
\hline $\mathbf{s 1 5 8 5 0}$ & 3906 & $\mathbf{8 0 9 0}$ & $\mathbf{8 0 4 2}$ & 8042 \\
\hline $\mathbf{s 3 8 4 1 7}$ & $\mathbf{2 1 8 7 9}$ & $\mathbf{2 9 2 8 2}$ & 21829 & 22085 \\
\hline $\mathbf{s 3 8 5 8 4}$ & 15522 & $\mathbf{2 0 5 7 0}$ & $\mathbf{2 0 5 0 9}$ & 20509 \\
\hline \hline
\end{tabular}

TABLE V

PBO VERSUS SIM RESULTS WITH AT MOST 10 INPUT FLIPS

\begin{tabular}{l|r|r|r|r}
\hline \hline \multirow{2}{*}{$T$} & \multicolumn{2}{|c|}{ PBO } & \multicolumn{2}{c}{ SIM } \\
\cline { 2 - 5 } $\mathbf{c 4 3 2}$ & $1000 s$ & $10000 s$ & $1000 s$ & $10000 s$ \\
\hline $\mathbf{c 4 9 9}$ & $\mathbf{9 2 1}$ & $\mathbf{9 9 6}$ & 822 & 822 \\
\hline $\mathbf{1 4 8 0}$ & $\mathbf{1 9 1 2}$ & 2306 & 1893 & 1893 \\
\hline $\mathbf{c 1 3 5 5}$ & $\mathbf{1 6 0 4}$ & $\mathbf{1 6 3 4}$ & 1342 & 1358 \\
\hline $\mathbf{c 1 9 0 8}$ & $\mathbf{2 1 4 5}$ & $\mathbf{2 1 9 7}$ & 1751 & 1769 \\
\hline $\mathbf{c 2 6 7 0}$ & $\mathbf{1 6 8 8}$ & $\mathbf{1 8 0 8}$ & 1535 & 1568 \\
\hline $\mathbf{c 3 5 4 0}$ & 4486 & 5175 & $\mathbf{5 3 7 2}$ & $\mathbf{5 3 7 2}$ \\
\hline $\mathbf{c 5 3 1 5}$ & 3337 & $\mathbf{4 9 8 5}$ & $\mathbf{3 9 7 7}$ & 3984 \\
\hline $\mathbf{c 6 2 8 8}$ & 87107 & 100683 & $\mathbf{1 1 1 2 6 8}$ & $\mathbf{1 1 1 2 6 8}$ \\
\hline $\mathbf{c 7 5 5 2}$ & 6790 & $\mathbf{8 8 9 5}$ & $\mathbf{7 7 6 6}$ & 7766 \\
\hline \hline $\mathbf{s 7 1 3}$ & $\mathbf{1 5 5 4}$ & $\mathbf{1 6 1 5}$ & 1513 & 1602 \\
\hline $\mathbf{s 1 2 3 8}$ & $\mathbf{7 7 3}$ & 792 & 766 & $\mathbf{8 2 8}$ \\
\hline $\mathbf{s 1 4 2 3}$ & $\mathbf{2 4 7 8}$ & $\mathbf{3 4 6 5}$ & 1644 & 1966 \\
\hline $\mathbf{s 9 2 3 4}$ & 4827 & $\mathbf{6 3 4 9}$ & $\mathbf{5 3 3 7}$ & 5842 \\
\hline $\mathbf{s 1 3 2 0 7}$ & 5888 & $\mathbf{1 0 5 3 0}$ & $\mathbf{8 1 7 4}$ & 8678 \\
\hline $\mathbf{s 1 5 8 5 0}$ & 4049 & 4049 & $\mathbf{8 0 0 8}$ & $\mathbf{8 0 0 8}$ \\
\hline $\mathbf{s 3 8 4 1 7}$ & $\mathbf{2 1 1 5 4}$ & $\mathbf{2 1 1 5 4}$ & 19777 & 20556 \\
\hline $\mathbf{s 3 8 5 8 4}$ & 16044 & 16044 & $\mathbf{1 9 3 4 3}$ & $\mathbf{1 9 8 9 0}$ \\
\hline \hline
\end{tabular}

ultimate time-out has been reached.

Next, instead of attempting to characterize the reachable states of ISCAS89 circuits as input constraints, we have implemented the non-trivial Hamming distance input constraints, as described in Section VII, as a proof of the applicability and effectiveness of input constraints using PBO. Obtaining a realistic set of valid states for the ISCAS89 circuits is beyond the scope of this work. Table $\mathrm{V}$ shows the results of PBO versus SIM with a unit-delay model, constrained to at most $d=10$ primary input flips, for the ISCAS benchmarks that have at least 10 primary inputs. The resulting activities are expectedly generally lower than those in Table I because of the added restrictions on input switching. Fig. 12 plots the PBO versus SIM activities with input constraints of at most $d=10$ bit flips on a logarithmic scale. Again, our PBO-based improves with increasing time-outs. After 10000 seconds, PBO generates $10 \%$ higher activities than SIM on average.

\section{Conclusion}

This work proposes a pseudo-Boolean satisfiability-based framework for finding the input sequence that maximizes single-cycle circuit activity. The method is extended to take into account multiple gate transitions during a clock-cycle. The integration of various external input constraints into the symbolic problem formulation is also described. Several optimizations are presented, such as the grouping of gates that are likely to switch in tandem into switching equivalence classes. The experimental results on ISCAS benchmarks show $7 \%$ higher activities on average compared to parallel-pattern random simulations within 10000 seconds, and further improvements with longer time-outs.

The theory and results of this paper confirm the need for further research in satisfiability-based solutions for problems in low-power design, especially given the tremendous rate of advancement in satisfiability engines and their extensions.

\section{REFERENCES}

[1] P. M. Morgado, P. F. Flores and L. M. Silveira, "Generating realistic stimuli for accurate power grid analysis," ACM TODAES, Vol. 14, No. 3, pp. 1-26, May 2009. 
[2] M. Xakellis and F. Najm, "Statistical estimation of the switching activity in digital circuits," IEEE DAC, pp. 728-733, 1994.

[3] F. Najm, "A survey of power estimation techniques in VLSI circuits," IEEE Trans. on VLSI, Vol. 2, No. 4, pp. 446-455, Dec. 1994.

[4] H. Kriplani, F. Najm and I. Hajj, "Maximum current estimation in CMOS circuits," IEEE DAC, pp. 2-7, 1992.

[5] A. T. Freitas, H. C. Neto and A. L. Oliveira, "On the complexity of power estimation problems," ILWS, pp. 176-181, 2004.

[6] N. E. Evmorfopoulos, G. I. Stamoulis and J. N. Avaritsiotis, "A Monte Carlo approach for maximum power estimation based on extreme value theory", IEEE trans. On CAD, Vol. 21, No. 4, April 2002, pp 415-432.

[7] H. Kriplani, F. Najm, P. Yang and I. Hajj, "Resolving signal correlations for estimating maximum currents in CMOS combinational circuits," IEEE DAC, pp. 2-7, 1993.

[8] C.-T. Hsieh, J.-C. Lin and S.-C. Chang, "A vectorless estimation of maximum instantaneous current for sequential circuits," IEEE ICCAD, pp. 537-540, Nov. 2004.

[9] C.-Y Wang and K. Roy, "Maximum power estimation for CMOS circuits using deterministic and statistical approaches," IEEE Trans. on VLSI, Vol. 6, pp. 134-140, 1998.

[10] C.-Y Wang, K. Roy, "Estimation of maximum power for sequential circuits considering spurious transitions," IEEE ICCD, pp. 746-751, 1997.

[11] C.-Y Wang, K. Roy, "COSMOS: a continuous optimization approach for maximum power estimation of CMOS circuits," IEEE ICCAD, pp. 52-55, 1997.

[12] M. S. Hsiao, E. M Rudnick and J. H. Patel, "Effects of delay models on peak power estimation of VLSI sequential circuits," IEEE ICCAD, pp. 45-51, Nov. 1997.

[13] M. S. Hsiao, "Peak power estimation using genetic spot optimization for large VLSI circuits," IEEE DATE, pp. 175-179, Nov. 1999.

[14] Q. Wu , Q. Qiu and M. Pedram, "Estimation of peak power dissipation in VLSI circuits using the limiting distributions of extreme order statistics," IEEE Trans. on CAD, Vol. 20, No. 8, pp. 942-956, Aug. 2001.

[15] S. Devadas, K. Keutzer and J. White, "Estimation of power dissipation in CMOS combinational circuits using boolean function manipulation," IEEE Trans. on CAD, pp. 373-383, Mar. 1992.

[16] P. M. Morgado, P. F. Flores, J. C. Monteiro and L. M. Silveira, "Generating worst-case stimuli for accurate power grid analysis," PATMOS, pp. 247-257, Sep. 2008.

[17] H. Mangassarian, A. Veneris, S. Safarpour, F. N. Najm and M. S. Abadir, "Maximum circuit activity estimation using pseudo-Boolean satisfiability," DATE, pp. 1538-1543, Apr. 2007.

[18] J. P. Marques-Silva and K. A. Sakallah, "GRASP - A search algorithm for propositional satisfiability," IEEE Trans. on comput., Vol. 48, No. 5, pp. 506-521, May 1999.

[19] M. H. Moskewicz, C. F. Madigan, Y. Zhao and L. Zhang, "Chaff: Engineering an efficient SAT solver," IEEE DAC, pp. 530-535, jun. 2001.

[20] N. Eén and N. Sörensson, "An extensible SAT-solver," SAT, pp. 502-518, 2003.

[21] T. Larrabee, "Test pattern generation using-Boolean satisfiability," IEEE Trans. on CAD, Vol. 11, No. 1, pp. 4-15, 1992.

[22] N. Eén and N. Sörensson, "Translating pseudo-boolean constraints into SAT," JSAT, Vol. 2, pp. 1-26, 2006.

[23] F. Aloul, A. Ramani, I. Markov and K. Sakallah, "PBS: A backtrack search pseudo-Boolean solver," SAT, 2002.

[24] H. Sheini and K. Sakallah, "Pueblo: A hybrid pseudo-boolean SAT solver," JSAT, Vol. 2, pp. 157-181, 2006.

[25] R. Drechsler, Advanced Formal Verification. Kluwer Academic Publishers, 2004.

[26] A. Smith, A. Veneris, M. F. Ali, and A. Viglas, "Fault diagnosis and logic debugging using Boolean satisfiability," IEEE Trans. on CAD, Vol. 24, No. 10, pp. 1606-1621, 2005.

[27] R. G. Wood and R. A. Rutenbar, "FPGA routing and routability estimation via Boolean satisfiability," IEEE Trans. on VLSI, Vol. 6, No. 1, pp. 222-231, Jun. 1998.

[28] M. Davis, G. Logemann and D. Loveland, "A machine program for theorem proving," Comm. of ACM, Vol. 5, No. 7, pp. 394-397, 1962

[29] O. Bailleux and Y. Boufkhad, "Efficient CNF encoding of Boolean cardinality constraints," $C P$, Vol. 2833, LNCS, 2003.

[30] J. Marques-Silva, I. Lynce and S. Malik, "Conflict-Driven Clause Learning SAT Solvers", Handbook of Satisfiability, IOS Press, pp. 131153, 2009.

[31] O. Roussel and V. Manquinho, "Pseudo-Boolean and Cardinality Constraints", Handbook of Satisfiability, IOS Press, pp. 695-733, 2009.

[32] V. Manquinho and J. Marques-Silva, "On Using Cutting Planes in Pseudo-Boolean Optimization”, JSAT 2(1-4), pp. 209-219, 2006.
[33] L. Guerra E Silva, J. Marques-Silva, L. M. Silveira and K. Sakallah, "Satisfiability Models and Algorithms for Circuit Delay Computation," ACM Trans. Des. Autom. Electron. Syst., Vol. 7, No. 1, pp. 137-158, Jan. 2002.

[34] R. Drechsler, Advanced Formal Verification. Kluwer Academic Publishers, 2004.

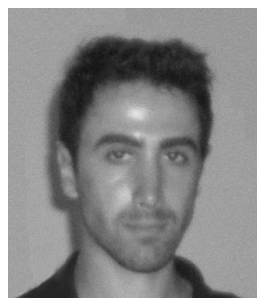

Hratch Mangassarian (S'01) received the B.E. degree in Computer and Communications Engineering with high distinction from the American University of Beirut in 2005, and the M.A.Sc. degree in Electrical and Computer Engineering from the University of Toronto in 2008, where he is currently working toward the Ph.D. degree. His research is on formal verification and automated design debugging of digital designs, as well as QBF and its applications to CAD.

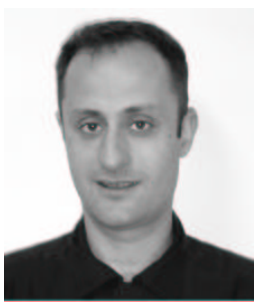

Andreas Veneris (S'96-M'99-SM'05) received a Diploma in Computer Engineering and Informatics from the University of Patras in 1991, an M.S. degree in Computer Science from the University of Southern California, Los Angeles in 1992 and a Ph.D. degree in Computer Science from the University of Illinois at Urbana-Champaign in 1998. In 1998 he was a visiting faculty at the University of Illinois until 1999 when he joined the Department of Electrical and Computer Engineering and the Department of Computer Science at the University of Toronto where today he is a Professor. His research interests include CAD for debugging, verification, synthesis and test of digital circuits/systems, and combinatorics. He has received several teaching awards, a best paper award and two best paper nominations. He is the author of one book and he holds three patents.

$\mathrm{He}$ is a member of ACM, IEEE, AAAS, Technical Chamber of Greece, Professional Engineers of Ontario and The Planetary Society

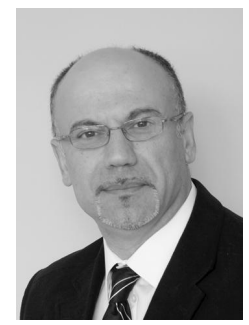

Farid N. Najm (S'85-M'89-SM'96-F'03) is a Professor in the department of electrical and computer engineering (ECE) at the University of Toronto, Ontario, Canada. He received the B.E. degree in electrical engineering from the American University of Beirut in 1983 and the Ph.D. degree in ECE from the University of Illinois at Urbana-Champaign (UIUC) in 1989. From 1989 to 1992, he worked with Texas Instruments, Dallas, Texas. He then joined the ECE department at UIUC as an Assistant Professor and became Associate Professor in 1997. In 1999, he joined the ECE department at the University of Toronto, where he is now Professor and Chair.

Dr. Najm is a Fellow of the IEEE and of the Canadian Academy of Engineering (CAE). He was Associate Editor for the IEEE TRANSACTIONS ON COMPUTER-AIDED DESIGN OF INTEGRATED CIRCUITS AND SySTEMS from 2001 to 2009. He has received an IEEE Transactions on CAD Best Paper Award, an NSF Research Initiation Award, an NSF CAREER Award, and was an Associate Editor for the IEEE TRANSACTIONS ON VERY LARGE SCALE InTEgRation (VLSI) Systems from 1997 to 2002. He serves on the executive committee of the International Symposium on Low-Power Electronics and Design (ISLPED), and has served on the technical committees of various conferences, including ICCAD, DAC, CICC, ISQED, and ISLPED. In 2010, Dr. Najm authored the book Circuit Simulation (John Wiley and Sons, New York). His research is on CAD for VLSI, with an emphasis on circuit level issues related to power, timing, variability, and reliability. 